Appl. Math. J. Chinese Univ.

2018, 33(4): 436-467

\title{
Novel applications of bipolar single-valued neutrosophic competition graphs
}

\author{
Muhammad Akram ${ }^{1} \quad$ Maryam Nasir $^{1} \quad$ K. P. Shum ${ }^{2}$
}

\begin{abstract}
Bipolar single-valued neutrosophic models are the generalization of bipolar fuzzy models. We first introduce the concept of bipolar single-valued neutrosophic competition graphs. We then, discuss some important propositions related to bipolar single-valued neutrosophic competition graphs. We define bipolar single-valued neutrosophic economic competition graphs and $m$-step bipolar single-valued neutrosophic economic competition graphs. Further, we describe applications of bipolar single-valued neutrosophic competition graphs in organizational designations and brands competition. Finally, we present our improved methods by algorithms.
\end{abstract}

\section{$\S 1 \quad$ Introduction}

The notion of competition graphs was introduced by Cohen [11] in 1968, depending upon a problem in ecology. The competition graphs have many utilizations in solving daily life problems, including channel assignment, modeling of complex economic, phytogenetic tree reconstruction, coding and energy systems.

Fuzzy set theory [34] and intuitionistic fuzzy sets theory [9] are useful models for dealing with uncertainty and incomplete information. But they may not be sufficient in modeling of indeterminate and inconsistent information encountered in real world. In order to cope with this issue, neutrosophic set theory was proposed by Smarandache [20] as a generalization of fuzzy sets and intuitionistic fuzzy sets. However, since neutrosophic sets are identified by three functions called truth-membership $(t)$, indeterminacy-membership $(i)$ and falsity-membership $(f)$ whose values are real standard or non-standard subset of unit interval $] 0^{-}, 1^{+}[$. There are some difficulties in modeling of some problems in engineering and sciences. To overcome these difficulties, The single valued neutrosophic set was introduced for the first time by Smarandache [21] in 1998 and later by Wang et al. [25]. Ye [27, 28, 29, 30, 31, 32] has proposed the concepts of a single valued neutrosophic graph, a single valued neutrosophic tree, a minimum spanning tree, and

Received: 2017-04-05. Revised: 2018-05-22.

MR Subject Classification: 03E72, 68R10, 68R05.

Keywords: bipolar single-valued neutrosophic digraphs, $m$-step bipolar single-valued neutrosophic economic competition graphs, algorithm.

Digital Object Identifier(DOI): https://doi.org/10.1007/s11766-018-3541-9. 
novel applications of neutrosophic sets.

Deli et al. [12] extended the ideas of bipolar fuzzy sets [36] and neutrosophic sets to bipolar neutrosophic sets and studied its operations and applications in decision making problems. Smarandache [23] proposed notion of neutrosophic graph and they separated them to four main categories. $\mathrm{Wu}[26]$ discussed fuzzy digraphs. The concept of fuzzy $k$-competition graphs and $p$-competition fuzzy graphs was first introduced by Smanta and Pal in [17], it was further studied in $[8,14,19]$. Akram and Shahzadi [5] first introduced the notion of neutrosophic soft graphs and gave its applications, it was further discussed in [3-6]. Samanta et al. [18] also introduced the concepts of fuzzy $m$-step neighbouthood graphs, fuzzy economic competition graphs, and $m$-step economic competitions graphs. Akram and Nasir [6] considered interval-valued neutrosophic competition graphs. All the predator-prey relations cannot only be represented by neutrosophic competition graphs. For example, a specie may have positive and negative truth-membership, indeterminacy, and false-memberships at the same time. These are bipolar information which are neutrosophic in nature. This idea motivates the necessity of bipolar neutrosophic competition graphs. In this paper, we first introduce the concept of bipolar neutrosophic competition graphs. We then discuss the method of construction of bipolar neutrosophic competition graph of the Cartesian product of bipolar neutrosophic digraph. Further, we describe applications of bipolar neutrosophic competition graphs in organizational designations and brands competition. Finally, we present our improved methods by algorithms.

\section{$\S 2$ Bipolar Single-Valued Neutrosophic Competition Graphs}

Definition 2.1. [34, 35] A fuzzy set $\mu$ in a universe $X$ is a mapping $\mu: X \rightarrow[0,1]$. A fuzzy relation on $X$ is a fuzzy set $\nu$ in $X \times X$.

Definition 2.2. [36] A bipolar fuzzy set on a non-empty set $X$ has the form

$$
A=\left\{\left(x, \mu_{A}^{p}(x), \mu_{A}^{n}(x)\right): x \in X\right\}
$$

where, $\mu_{A}^{p}: X \rightarrow[0,1]$ and $\mu_{A}^{n}: X \rightarrow[-1,0]$ are mappings. The positive membership value $\mu_{A}^{p}(x)$ represents the strength of truth or satisfaction of an element $x$ to a certain property corresponding to bipolar fuzzy set $A$ and $\mu_{A}^{n}(x)$ denotes the strength of satisfaction of an element $x$ to some counter property of bipolar fuzzy set $A$. If $\mu_{A}^{p}(x) \neq 0$ and $\mu_{A}^{n}(x)=0$ it is the situation when $x$ has only truth satisfaction degree for property $A$. If $\mu_{A}^{n}(x) \neq 0$ and $\mu_{A}^{p}(x)=0$, it is the case that $x$ is not satisfying the property of $A$ but satisfying the counter property to $A$. It is possible for $x$ that $\mu_{A}^{p}(x) \neq 0$ and $\mu_{A}^{n}(x) \neq 0$ when $x$ satisfies the property of $A$ as well as its counter property in some part of $X$.

Definition 2.3. [1] Let $X$ be a non-empty set. A mapping $B=\left(\mu_{B}^{p}, \mu_{B}^{n}\right): X \times X \rightarrow$ $[0,1] \times[-1,0]$ is a bipolar fuzzy relation on $X$ such that $\mu_{B}^{p}(x y) \in[0,1]$ and $\mu_{B}^{n}(x y) \in[-1,0]$ for $x, y \in X$.

Definition 2.4. [1] A bipolar fuzzy graph on $X$ is a pair $G=(A, B)$ where $A=\left(\mu_{A}^{p}, \mu_{A}^{n}\right)$ is a bipolar fuzzy set on $X$ and $B=\left(\mu_{B}^{p}, \mu_{B}^{n}\right)$ is a bipolar fuzzy relation in $X$ such that 


$$
\mu_{B}^{p}(x y) \leq \mu_{A}^{p}(x) \wedge \mu_{A}^{p}(y) \text { and } \mu_{B}^{n}(x y) \geq \mu_{A}^{n}(x) \vee \mu_{A}^{n}(y) \text { for all } x, y \in X .
$$

Definition 2.5. [24] A neutrosophic set $A$ on a non-empty set $X$ is characterized by a truthmembership fuction $t_{A}: X \rightarrow[0,1]$, indeterminacy-membership function $i_{A}: X \rightarrow[0,1]$ and a falsity-membership function $f_{A}: X \rightarrow[0,1]$. There is no restriction on the sum of $t_{A}(x), i_{A}(x)$ and $f_{A}(x)$ for all $x \in X$.

Definition 2.6. [12] A bipolar single-valued neutrosophic set $A$ on a non-empty set $X$ is an object of the form

$$
A=\left\{\left(x, t_{A}^{p}(x), i_{A}^{p}(x), f_{A}^{p}(x), t_{A}^{n}(x), i_{A}^{n}(x), f_{A}^{n}(x)\right): x \in X\right\},
$$

where $t_{A}^{p}, i_{A}^{p}, f_{A}^{p}: X \rightarrow[0,1]$ and $t_{A}^{n}, i_{A}^{n}, f_{A}^{n}: X \rightarrow[-1,0]$. The positive values $t_{A}^{p}(x)$, $i_{A}^{p}(x), f_{A}^{p}(x)$ denote respectively the truth, indeterminacy and false-memberships degrees of an element $x \in X$, whereas, $t_{A}^{n}(x), i_{A}^{n}(x), f_{A}^{n}(x)$ denote the implicit counter property of the truth,indeterminacy and false-memberships degrees of the element $x \in X$ corresponding to the bipolar single-valued neutrosophic set $A$.

We now define bipolar single-valued neutrosophic digraph.

Definition 2.7. A bipolar single-valued neutrosophic digraph on a non-empty set $X$ is a pair $G=(A, \vec{B})$, (in short, $G$ ), where $A$ is a bipolar single-valued neutrosophic set on $X$ and $B$ is a bipolar single-valued neutrosophic relation on $X$, such that:

1. $t_{B}^{p} \overrightarrow{(x, y)} \leq t_{A}^{p}(x) \wedge t_{A}^{p}(y)$

2. $i_{B}^{p} \overrightarrow{(x, y)} \leq i_{A}^{p}(x) \wedge i_{A}^{p}(y)$

3. $f_{B}^{p} \overrightarrow{(x, y)} \leq f_{A}^{p}(x) \vee f_{A}^{p}(y)$,
4. $t_{B}^{n} \overrightarrow{(x, y)} \geq t_{A}^{n}(x) \vee t_{A}^{n}(y)$,

5. $i_{B}^{n} \overrightarrow{(x, y)} \geq i_{A}^{n}(x) \vee i_{A}^{n}(y)$

6. $f_{B}^{n} \overrightarrow{(x, y)} \geq f_{A}^{n}(x) \wedge f_{A}^{n}(y), \quad$ for all $x, y \in X$.

Example 2.1. We construct a bipolar single-valued neutrosophic digraph $G=(A, \vec{B})$ on $X=\{a, b, c\}$ as shown in Fig.1.

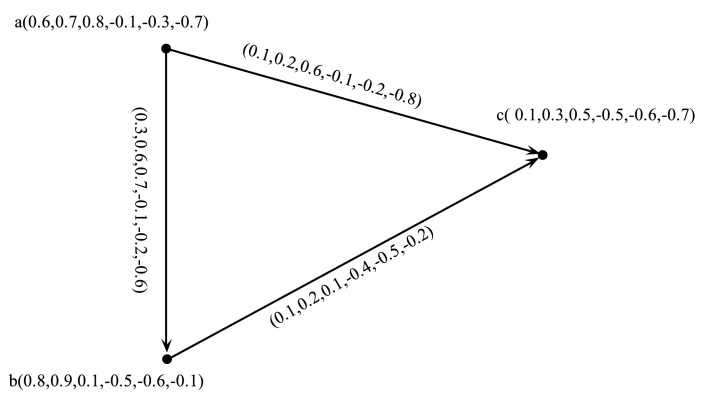

Figure 1: Bipolar single-valued neutrosophic digraph 
Definition 2.8. Let $\vec{G}$ be a bipolar single-valued neutrosophic digraph then bipolar singlevalued neutrosophic out-neighbourhoods of a vertex $x$ is a bipolar single-valued neutrosophic set

$$
\mathcal{N}^{+}(x)=\left(X_{x}^{+}, t_{x}^{(p)^{+}}, i_{x}^{(p)^{+}}, f_{x}^{(p)^{+}}, t_{x}^{(n)^{+}}, i_{x}^{(n)^{+}}, f_{x}^{(n)^{+}}\right)
$$

where,

$$
X_{x}^{+}=\left\{y \mid B_{1}^{p} \overrightarrow{(x, y)}>0, B_{2}^{p} \overrightarrow{(x, y)}>0, B_{3}^{p}\left(\overrightarrow{x, y)}>0, B_{1}^{n} \overrightarrow{(x, y)}<0, B_{2}^{n} \overrightarrow{(x, y)}<0, B_{3}^{n} \overrightarrow{(x, y)}<0\right\},\right.
$$

such that $t_{x}^{(p)^{+}}: X_{x}^{+} \rightarrow[0,1]$, defined by $t_{x}^{(p)^{+}}(y)=B_{1}^{p} \overrightarrow{(x, y)}, i_{x}^{(p)^{+}}: X_{x}^{+} \rightarrow[0,1]$, defined by $i_{x}^{(p)^{+}}(y)=B_{2}^{p} \overrightarrow{(x, y)}, f_{x}^{(p)^{+}}: X_{x}^{+} \rightarrow[0,1]$, defined by $f_{x}^{(p)^{+}}(y)=B_{3}^{p} \overrightarrow{(x, y)}, t_{x}^{(n)^{+}}: X_{x}^{+} \rightarrow[-1,0]$, defined by $t_{x}^{(n)^{+}}(y)=B_{1}^{n} \overrightarrow{(x, y)}, i_{x}^{(n)^{+}}: X_{x}^{+} \rightarrow[-1,0]$, defined by $i_{x}^{(n)^{+}}(y)=B_{2}^{n} \overrightarrow{(x, y)}, f_{x}^{(n)^{+}}$: $X_{x}^{+} \rightarrow[-1,0]$, defined by $f_{x}^{(n)^{+}}(y)=B_{3}^{n} \overrightarrow{(x, y)}$.

Definition 2.9. Let $\vec{G}$ be a bipolar single-valued neutrosophic digraph then bipolar singlevalued neutrosophic in-neighbourhoods of a vertex $x$ is a bipolar single-valued neutrosophic set

$$
\mathcal{N}^{-}(x)=\left(X_{x}^{-}, t_{x}^{(p)^{-}}, i_{x}^{(p)^{-}}, f_{x}^{(p)^{-}}, t_{x}^{(n)^{-}}, i_{x}^{(n)^{-}}, f_{x}^{(n)^{-}}\right)
$$

where,

$$
X_{x}^{-}=\left\{y \mid B_{1}^{p} \overrightarrow{(y, x)}>0, B_{2}^{p} \overrightarrow{(y, x)}>0, B_{3}^{p} \overrightarrow{(y, x)}>0, B_{1}^{n} \overrightarrow{(y, x)}<0, B_{2}^{n} \overrightarrow{(y, x)}<0, B_{3}^{n} \overrightarrow{(y, x)}<0\right\},
$$

such that $t_{x}^{(p)^{-}}: X_{x}^{-} \rightarrow[0,1]$, defined by $t_{x}^{(p)^{-}}(y)=B_{1}^{p} \overrightarrow{(y, x)}, i_{x}^{(p)^{-}}: X_{x}^{-} \rightarrow[0,1]$, defined by $i_{x}^{(p)^{-}}(y)=B_{2}^{p}\left(\overrightarrow{y, x)}, f_{x}^{(p)^{-}}: X_{x}^{-} \rightarrow[0,1]\right.$, defined by $f_{x}^{(p)^{-}}(y)=B_{3}^{p}(y, x), t_{x}^{(n)^{-}}: X_{x}^{-} \rightarrow[-1,0]$, defined by $t_{x}^{(n)^{-}}(y)=B_{1}^{n} \overrightarrow{(y, x)}, i_{x}^{(n)^{-}}: X_{x}^{-} \rightarrow[-1,0]$, defined by $i_{x}^{(n)^{-}}(y)=B_{2}^{n} \overrightarrow{(y, x)}, f_{x}^{(n)^{-}}$: $X_{x}^{-} \rightarrow[-1,0]$, defined by $f_{x}^{(n)^{-}}(y)=B_{3}^{n} \overrightarrow{(y, x)}$.

Example 2.2. Consider a bipolar single-valued neutrosophic digraph $G=(A, \vec{B})$ on $X=$ $\{a, b, c\}$ as shown in Fig.1. We have Table 1 and Table 2 representing bipolar single-valued neutrosophic out and in-neighbourhoods, respectively.

Table 1: Bipolar single-valued neutrosophic out-neighbourhoods

\begin{tabular}{|l|l|}
\hline$x$ & $\mathcal{N}^{+}(x)$ \\
\hline $\mathrm{a}$ & $\{(\mathrm{b}, 0.3,0.6,0.7,-0.1,-0.2,-0.6),(\mathrm{c}, 0.1,0.2,0.6,-0.1,-0.2,-0.8)\}$ \\
$\mathrm{b}$ & $\{(\mathrm{c}, 0.1,0.2,0.1,-0.4,-0.5,-0.2)\}$ \\
$\mathrm{c}$ & $\emptyset$ \\
\hline
\end{tabular}

Table 2: Bipolar single-valued neutrosophic in-neighbourhoods

\begin{tabular}{|l|l|}
\hline$x$ & $\mathcal{N}^{-}(x)$ \\
\hline $\mathrm{a}$ & $\emptyset$ \\
$\mathrm{b}$ & $\{(\mathrm{a}, 0.3,0.6,0.7,-0.1,-0.2,-0.6)\}$ \\
$\mathrm{c}$ & $\{(\mathrm{a}, 0.1,0.2,0.6,-0.1,-0.2,-0.8),(\mathrm{b}, 0.1,0.2,0.1,-0.4,-0.5,-0.2)\}$ \\
\hline
\end{tabular}


Definition 2.10. The height of bipolar single-valued neutrosophic set $A=\left(t_{A}^{p}(x), i_{A}^{p}(x)\right.$, $\left.f_{A}^{p}(x), t_{A}^{n}(x), i_{A}^{n}(x), f_{A}^{n}(x)\right)$ in universe of discourse $X$ is defined as,

$$
\begin{aligned}
h(A) & =\left(h_{1}(A), h_{2}(A), h_{3}(A), h_{4}(A), h_{5}(A), h_{6}(A)\right) \\
& =\left(\sup _{x \in X} t_{A}^{p}(x), \sup _{x \in X} i_{A}^{p}(x), \inf _{x \in X} f_{A}^{p}(x), \sup _{x \in X} t_{A}^{p}(x), \sup _{x \in X} i_{A}^{p}(x), \inf _{x \in X} f_{A}^{p}(x)\right), \quad \text { for all } \quad x \in X .
\end{aligned}
$$

Example 2.3. The height of a bipolar single-valued neutrosophic set $A=\{(a, 0.5,0.7,0.2$, $-0.8,-0.9,-0.3),(b, 0.1,0.2,1,-0.5,-0.7,-0.6),(c, 0.3,0.5,0.3,-0.8,-0.6,-0.4)\}$ in $X=\{a, b, c\}$ is $h(A)=(0.5,0.7,0.2,0.5,0.7,0.2)$.

Definition 2.11. A bipolar single-valued neutrosophic competition graph of a bipolar singlevalued neutrosophic graph $\vec{G}=(A, \vec{B})$ is an undirected bipolar single-valued neutrosophic graph $\overrightarrow{\mathcal{C}(G)}=(A, R)$ which has the same vertex set as in $\vec{G}$ and there is an edge between two vertices $x$ and $y$ if and only if $\mathcal{N}^{+}(x) \cap \mathcal{N}^{+}(y)$ is non-empty. The positive truthmembership, indeterminacy-membership, falsity-membership and negative truth-membership, indeterminacy-membership, falsity-membership values of the edge $(x, y)$ are defined as,

1. $t_{R}^{p}(x, y)=\left(t_{A}^{p}(x) \wedge t_{A}^{p}(y)\right) h_{1}\left(\mathcal{N}^{+}(x) \cap \mathcal{N}^{+}(y)\right.$,

2. $i_{R}^{p}(x, y)=\left(i_{A}^{p}(x) \wedge i_{A}^{p}(y)\right) h_{2}\left(\mathcal{N}^{+}(x) \cap \mathcal{N}^{+}(y)\right.$,

3. $f_{R}^{p}(x, y)=\left(f_{A}^{p}(x) \vee f_{A}^{p}(y)\right) h_{3}\left(\mathcal{N}^{+}(x) \cap \mathcal{N}^{+}(y)\right.$,

4. $t_{R}^{n}(x, y)=\left(t_{A}^{n}(x) \vee t_{A}^{n}(y)\right) h_{4}\left(\mathcal{N}^{+}(x) \cap \mathcal{N}^{+}(y)\right.$,

5. $i_{R}^{n}(x, y)=\left(i_{A}^{n}(x) \vee i_{A}^{n}(y)\right) h_{5}\left(\mathcal{N}^{+}(x) \cap \mathcal{N}^{+}(y)\right.$,

6. $f_{R}^{n}(x, y)=\left(f_{A}^{n}(x) \wedge f_{A}^{n}(y)\right) h_{6}\left(\mathcal{N}^{+}(x) \cap \mathcal{N}^{+}(y)\right.$, for all $x, y \in X$.

Example 2.4. Consider $\vec{G}=(A, B)$ is a bipolar single-valued neutrosophic digraph on $X=$ $\{a, b, c\}$, such that $A=\{(a, 0.6,0.7,0.8,-0.1,-0.3,-0.7),(b, 0.8,0.9,0.1,-0.5,-0.6,-0.1),(c$, $0.1,0.3,0.5,-0.5,-0.6,-0.7)\}$, and $B=\{(\overrightarrow{a c}, 0.1,0.2,0.6,-0.1,-0.2,-0.8),(\overrightarrow{a b}, 0.3,0.6,0.7$, $-0.1,-0.2,-0.6),(\overrightarrow{b c}, 0.1,0.2,0.1,-0.4,-0.5,-0.2)\}$ as shown in Fig.2. By direct calculation-

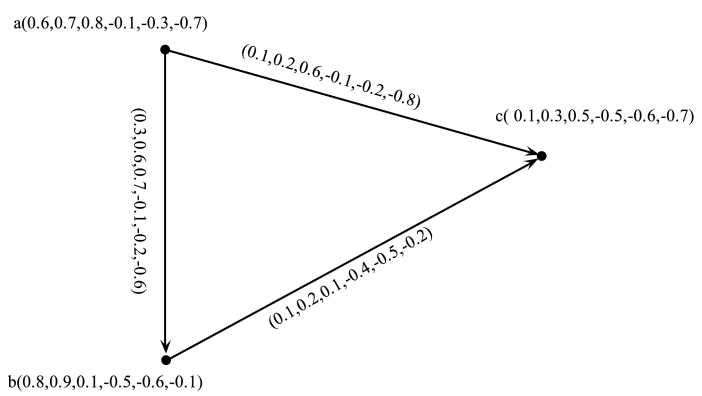

Figure 2: Bipolar single-valued neutrosophic digraph

s we have Table 3 and Table 4 representing bipolar single-valued neutrosophic out and inneighbourhoods, respectively. 
Table 3: Bipolar single-valued neutrosophic out-neighbourhoods

\begin{tabular}{|l|l|}
\hline$x$ & $\mathcal{N}^{+}(x)$ \\
\hline $\mathrm{a}$ & $\{(\mathrm{b}, 0.3,0.6,0.7,-0.1,-0.2,-0.6),(\mathrm{c}, 0.1,0.2,0.6,-0.1,-0.2,-0.8)\}$ \\
$\mathrm{b}$ & $\{(\mathrm{c}, 0.1,0.2,0.1,-0.4,-0.5,-0.2)\}$ \\
$\mathrm{c}$ & $\emptyset$
\end{tabular}

Table 4: Bipolar single-valued neutrosophic in-neighbourhoods

\begin{tabular}{|l|l|}
\hline$x$ & $\mathcal{N}^{-}(x)$ \\
\hline $\mathrm{a}$ & $\emptyset$ \\
$\mathrm{b}$ & $\{(\mathrm{a}, 0.3,0.6,0.7,-0.1,-0.2,-0.6)\}$ \\
$\mathrm{c}$ & $\{(\mathrm{a}, 0.1,0.2,0.6,-0.1,-0.2,-0.8),(\mathrm{b}, 0.1,0.2,0.1,-0.4,-0.5,-0.2)\}$ \\
\hline
\end{tabular}

The bipolar single-valued neutrosophic competition graph of Fig.2 is shown in Fig.3.

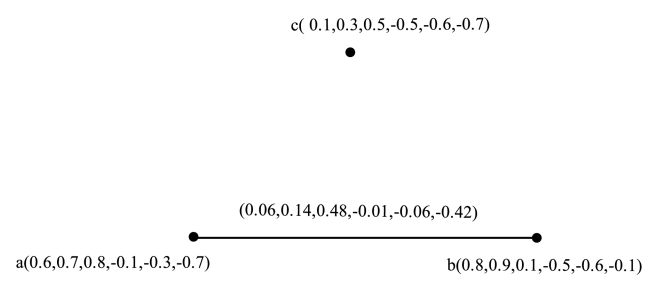

Figure 3: Bipolar single-valued neutrosophic competition graph

Definition 2.12. Consider a bipolar single-valued neutrosophic graph $G=(A, B)$, where $A=\left(A_{1}^{p}, A_{2}^{p}, A_{3}^{p}, A_{1}^{n}, A_{2}^{n}, A_{3}^{n}\right)$, and $B=\left(B_{1}^{p}, B_{2}^{p}, B_{3}^{p}, B_{1}^{n}, B_{2}^{n}, B_{3}^{n}\right)$ then, an edge $(x, y), x, y$ $\in X$ is called independent strong if

Otherwise, it is called weak.

$$
\begin{array}{ll}
\frac{1}{2}\left[A_{1}^{p}(x) \wedge A_{1}^{p}(y)\right]<B_{1}^{p}(x, y), & \frac{1}{2}\left[A_{1}^{n}(x) \vee A_{1}^{n}(y)\right]>B_{1}^{n}(x, y), \\
\frac{1}{2}\left[A_{2}^{p}(x) \wedge A_{2}^{p}(y)\right]<B_{2}^{p}(x, y), & \frac{1}{2}\left[A_{2}^{n}(x) \vee A_{2}^{n}(y)\right]>B_{2}^{n}(x, y), \\
\frac{1}{2}\left[A_{3}^{p}(x) \vee A_{3}^{p}(y)\right]>B_{3}^{p}(x, y), & \frac{1}{2}\left[A_{3}^{n}(x) \wedge A_{3}^{n}(y)\right]<B_{3}^{n}(x, y) .
\end{array}
$$

Theorem 2.1. Suppose $\vec{G}$ is a bipolar single-valued neutrosophic digraph. If $\mathcal{N}^{+}(x) \cap \mathcal{N}^{+}(y)$ contains only one element of $\vec{G}$, then the edge $(x, y)$ of $\mathcal{C}(\vec{G})$ is independent strong if and only if

$$
\begin{array}{rr}
\left|\left[\mathcal{N}^{+}(x) \cap \mathcal{N}^{+}(y)\right]\right|_{t^{p}}>0.5, & \left|\left[\mathcal{N}^{+}(x) \cap \mathcal{N}^{+}(y)\right]\right|_{t^{n}}<0.5 \\
\left|\left[\mathcal{N}^{+}(x) \cap \mathcal{N}^{+}(y)\right]\right|_{i^{p}}>0.5, & \left|\left[\mathcal{N}^{+}(x) \cap \mathcal{N}^{+}(y)\right]\right|_{i^{n}}<0.5 \\
\left|\left[\mathcal{N}^{+}(x) \cap \mathcal{N}^{+}(y)\right]\right|_{f^{p}}<0.5, & \left|\left[\mathcal{N}^{+}(x) \cap \mathcal{N}^{+}(y)\right]\right|_{f^{n}}<0.5 .
\end{array}
$$

Proof. Suppose, $\vec{G}$ is a bipolar single-valued neutrosophic digraph. Suppose $\mathcal{N}^{+}(x) \cap \mathcal{N}^{+}(y)=$ $\left(a, \breve{p^{p}}, q^{p}, r^{p}, \breve{p^{n}}, q^{n}, r^{n}\right)$, where, $\breve{p}^{p}, q^{p}, r^{p}, p^{n}, q^{n}, r^{n}$ are the positive truth-membership, 
indeterminacy-membership, falsity-membership and negative truth-membership, indeterminacymembership, falsity-membership values of either the edge $(x, a)$ or the edge $(y, a)$, respectively. Here,

$$
\begin{aligned}
& \left|\left[\mathcal{N}^{+}(x) \cap \mathcal{N}^{+}(y)\right]\right|_{t^{p}}=\breve{p}^{p}=h_{1}\left(\mathcal{N}^{+}(x) \cap \mathcal{N}^{+}(y)\right), \\
& \left|\left[\mathcal{N}^{+}(x) \cap \mathcal{N}^{+}(y)\right]\right|_{t^{n}}=\breve{p}^{n}=h_{4}\left(\mathcal{N}^{+}(x) \cap \mathcal{N}^{+}(y)\right), \\
& \left|\left[\mathcal{N}^{+}(x) \cap \mathcal{N}^{+}(y)\right]\right|_{i^{p}}=q^{p}=h_{2}\left(\mathcal{N}^{+}(x) \cap \mathcal{N}^{+}(y)\right), \\
& \left|\left[\mathcal{N}^{+}(x) \cap \mathcal{N}^{+}(y)\right]\right|_{i^{n}}=q^{n}=h_{5}\left(\mathcal{N}^{+}(x) \cap \mathcal{N}^{+}(y)\right), \\
& \left|\left[\mathcal{N}^{+}(x) \cap \mathcal{N}^{+}(y)\right]\right|_{f^{p}}=r^{p}=h_{3}\left(\mathcal{N}^{+}(x) \cap \mathcal{N}^{+}(y)\right), \\
& \left|\left[\mathcal{N}^{+}(x) \cap \mathcal{N}^{+}(y)\right]\right|_{f^{n}}=r^{n}=h_{6}\left(\mathcal{N}^{+}(x) \cap \mathcal{N}^{+}(y)\right) .
\end{aligned}
$$

Then,

$$
\begin{array}{ll}
B_{1}^{p}(x, y)=\breve{p^{p}} \times\left[A_{1}^{p}(x) \wedge A_{1}^{p}(y)\right], & B_{1}^{n}(x, y)=\breve{p^{n}} \times\left[A_{1}^{n}(x) \vee A_{1}^{n}(y)\right], \\
B_{2}^{p}(x, y)=q^{p} \times\left[A_{2}^{p}(x) \wedge A_{2}^{p}(y)\right], & B_{2}^{n}(x, y)=q^{n} \times\left[A_{2}^{n}(x) \vee A_{2}^{n}(y)\right], \\
B_{3}^{p}(x, y)=r^{p} \times\left[A_{3}^{p}(x) \vee A_{3}^{p}(y)\right], & B_{3}^{n}(x, y)=r^{n} \times\left[A_{3}^{n}(x) \wedge A_{3}^{n}(y)\right] .
\end{array}
$$

Therefore, the edge $(x, y)$ in $\mathcal{C}(\vec{G})$ is independent strong if and only if $\breve{p}^{p}>0.5, q^{p}>0.5$, $r^{p}<0.5, \breve{p^{n}}<0.5, q^{n}<0.5, r^{n}<0.5$, Hence, the edge $(x, y)$ of $\mathcal{C}(\vec{G})$ is independent strong if and only if

$$
\begin{array}{ll}
\left|\left[\mathcal{N}^{+}(x) \cap \mathcal{N}^{+}(y)\right]\right|_{t^{p}}>0.5, & \left|\left[\mathcal{N}^{+}(x) \cap \mathcal{N}^{+}(y)\right]\right|_{t^{n}}<0.5, \\
\left|\left[\mathcal{N}^{+}(x) \cap \mathcal{N}^{+}(y)\right]\right|_{i^{p}}>0.5, & \left|\left[\mathcal{N}^{+}(x) \cap \mathcal{N}^{+}(y)\right]\right|_{i^{n}}<0.5, \\
\left|\left[\mathcal{N}^{+}(x) \cap \mathcal{N}^{+}(y)\right]\right|_{f^{p}}<0.5, & \left|\left[\mathcal{N}^{+}(x) \cap \mathcal{N}^{+}(y)\right]\right|_{f^{n}}<0.5 .
\end{array}
$$

We illustrate the theorem with an example as shown in Fig.4.

Theorem 2.2. If all the edges of a bipolar single-valued neutrosophic digraph $\vec{G}$ are independent strong, then

$$
\begin{array}{ll}
\frac{B_{1}^{p}(x, y)}{\left(A_{1}^{p}(x) \wedge A_{1}^{p}(y)\right)^{2}}>0.5, & \frac{B_{1}^{n}(x, y)}{\left(A_{1}^{n}(x) \vee A_{1}^{n}(y)\right)^{2}}<0.5, \\
\frac{B_{2}^{p}(x, y)}{\left(A_{2}^{p}(x) \wedge A_{2}^{p}(y)\right)^{2}}>0.5, & \frac{B_{2}^{n}(x, y)}{\left(A_{2}^{n}(x) \vee A_{2}^{n}(y)\right)^{2}}<0.5, \\
\frac{B_{3}^{p}(x, y)}{\left(A_{3}^{p}(x) \vee A_{3}^{p}(y)\right)^{2}}<0.5, & \frac{B_{3}^{n}(x, y)}{\left(A_{3}^{n}(x) \wedge A_{3}^{n}(y)\right)^{2}}<0.5,
\end{array}
$$

for all edges $(x, y)$ in $\mathcal{C}(\vec{G})$.

Proof. Suppose all the edges of bipolar single-valued neutrosophic digraph $\vec{G}$ are independent 


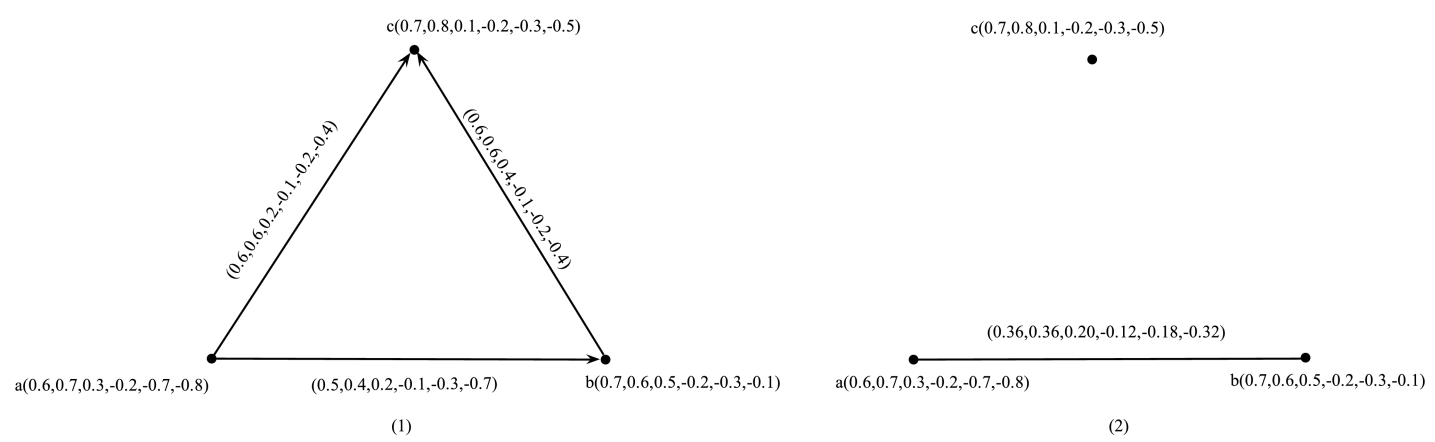

Figure 4: Bipolar single-valued neutrosophic competition graph (1) Bipolar single-valued neutrosophic digraph (2) Corresponding bipolar single-valued neutrosophic competition graph

strong. Then

$$
\begin{array}{ll}
\frac{1}{2}\left[A_{1}^{p}(x) \wedge A_{1}^{p}(y)\right]<B_{1}^{p}(\overrightarrow{x, y)}, & \frac{1}{2}\left[A_{1}^{n}(x) \vee A_{1}^{n}(y)\right]>B_{1}^{n} \overrightarrow{(x, y)}, \\
\frac{1}{2}\left[A_{2}^{p}(x) \wedge A_{2}^{p}(y)\right]<B_{2}^{p} \overrightarrow{(x, y)}, & \frac{1}{2}\left[A_{2}^{n}(x) \vee A_{2}^{n}(y)\right]>B_{2}^{n} \overrightarrow{(x, y)}, \\
\frac{1}{2}\left[A_{3}^{p}(x) \vee A_{3}^{p}(y)\right]>B_{3}^{p} \overrightarrow{(x, y)}, & \frac{1}{2}\left[A_{3}^{n}(x) \wedge A_{3}^{n}(y)\right]<B_{3}^{n} \overrightarrow{(x, y)},
\end{array}
$$

for all the edges $(x, y)$ in $\vec{G}$. Let the corresponding bipolar single-valued neutrosophic competition graph be $\mathcal{C}(\vec{G})$. Then their arise two cases:

Case(1). When $\mathcal{N}^{+}(x) \cap \mathcal{N}^{+}(y)=\emptyset$ for all $x, y \in X$. Then there exists no edge in $\mathcal{C}(\vec{G})$ between $x$ and $y$. Thus, we have nothing to prove in this case.

$\operatorname{Case}(2)$. When $\mathcal{N}^{+}(x) \cap \mathcal{N}^{+}(y) \neq \emptyset$. Let $\mathcal{N}^{+}(x) \cap \mathcal{N}^{+}(y)=\left\{\left(a_{1}, m_{1}^{p}, r_{1}^{p}, \breve{p}_{1}^{p}, m_{1}^{n}, r_{1}^{n}, \breve{p}_{1}^{n}\right),\left(a_{2}\right.\right.$, $\left.\left.m_{2}^{p}, r_{2}^{p}, \breve{p}_{2}^{p}, m_{2}^{n}, r_{2}^{n}, \breve{p}_{2}^{n}\right), \ldots,\left(a_{l}, m_{l}^{p}, r_{l}^{p}, \breve{p}_{l}^{p}, m_{l}^{n}, r_{l}^{n}, \breve{p}_{l}^{n}\right)\right\}$, where $m_{i}^{p}, r_{i}^{p}, \breve{p}_{i}^{p}, m_{i}^{n}, r_{i}^{n}, \breve{p}_{i}^{n}$ are the positive and negative truth-membership, indeterminacy-membership and falsity-membership values of either $\overrightarrow{\left(x, a_{i}\right)}$ or $\overrightarrow{\left(y, a_{i}\right)}$ for $i=1,2, \ldots, l$, respectively. Thus,

$$
\begin{aligned}
m_{i}^{p} & =\left[B_{1}^{p} \overrightarrow{\left(x, a_{i}\right)} \wedge B_{1}^{p} \overrightarrow{\left(y, a_{i}\right)}\right], & m_{i}^{n}=\left[B_{1}^{n} \overrightarrow{\left(x, a_{i}\right)} \vee B_{1}^{n} \overrightarrow{\left(y, a_{i}\right)}\right], \\
r_{i}^{p} & =\left[B_{2}^{p} \overrightarrow{\left(x, a_{i}\right)} \wedge B_{2}^{p} \overrightarrow{\left(y, a_{i}\right)}\right], & r_{i}^{n}=\left[B_{2}^{n} \overrightarrow{\left(x, a_{i}\right)} \vee B_{2}^{n} \overrightarrow{\left(y, a_{i}\right)}\right], \\
\breve{p}_{i}^{p} & =\left[B_{3}^{p} \overrightarrow{\left(x, a_{i}\right)} \vee B_{3}^{p} \overrightarrow{\left(y, a_{i}\right)}\right], & \breve{p}_{i}^{n}=\left[B_{3}^{n} \overrightarrow{\left(x, a_{i}\right)} \vee B_{3}^{n} \overrightarrow{\left(y, a_{i}\right)}\right], \quad \text { for } \quad i=1,2, \ldots, l .
\end{aligned}
$$

Suppose,

$$
\begin{aligned}
& h_{1}\left(\mathcal{N}^{+}(x) \cap \mathcal{N}^{+}(y)\right)=\max \left\{m_{i}^{p}, \quad i=1,2, \ldots, l\right\}=m_{\max }^{p}, \\
& h_{2}\left(\mathcal{N}^{+}(x) \cap \mathcal{N}^{+}(y)\right)=\max \left\{r_{i}^{p}, \quad i=1,2, \ldots, l\right\}=r_{\max }^{p}, \\
& h_{3}\left(\mathcal{N}^{+}(x) \cap \mathcal{N}^{+}(y)\right)=\min \left\{\breve{p}_{i}^{p}, \quad i=1,2, \ldots, l\right\}=\breve{p}_{\min }^{p}, \\
& h_{4}\left(\mathcal{N}^{+}(x) \cap \mathcal{N}^{+}(y)\right)=\min \left\{m_{i}^{n}, \quad i=1,2, \ldots, l\right\}=m_{\max }^{n}, \\
& h_{5}\left(\mathcal{N}^{+}(x) \cap \mathcal{N}^{+}(y)\right)=\min \left\{r_{i}^{n}, \quad i=1,2, \ldots, l\right\}=r_{\max }^{n}, \\
& h_{6}\left(\mathcal{N}^{+}(x) \cap \mathcal{N}^{+}(y)\right)=\min \left\{\breve{p}_{i}^{n}, \quad i=1,2, \ldots, l\right\}=\breve{p}_{\min }^{n} .
\end{aligned}
$$

Obviously, $\quad m_{\max }^{p}>B_{1}^{p}\left(\overrightarrow{x, y)}, r_{\max }^{p}>B_{2}^{p} \overrightarrow{(x, y)}, \breve{p}_{\min }^{p}<B_{3}^{p} \overrightarrow{(x, y)}, m_{\max }^{n}<B_{1}^{n} \overrightarrow{(x, y)}\right.$, 
$r_{\max }^{n}<B_{2}^{n} \overrightarrow{(x, y)}, \breve{p}_{\min }^{n}<B_{3}^{n} \overrightarrow{(x, y)}$ for all edges $\overrightarrow{(x, y)}$ shows that

$$
\begin{array}{ll}
\frac{m_{\max }^{p}}{A_{1}^{p}(x) \wedge A_{1}^{p}(y)}>\frac{B_{1}^{p} \overrightarrow{(x, y)}}{A_{1}^{p}(x) \wedge A_{1}^{p}(y)}>0.5, & \frac{m_{\max }^{n}}{A_{1}^{n}(x) \vee A_{1}^{n}(y)}<\frac{B_{1}^{n}(x, y)}{A_{1}^{n}(x) \vee A_{1}^{n}(y)}<0.5, \\
\frac{r_{\max }^{p}}{A_{2}^{p}(x) \wedge A_{2}^{p}(y)}>\frac{B_{2}^{p}(x, y)}{A_{2}^{p}(x) \wedge A_{2}^{p}(y)}>0.5, & \frac{r_{\max }^{n}}{A_{2}^{n}(x) \vee A_{2}^{n}(y)}<\frac{B_{2}^{n}(x, y)}{A_{2}^{n}(x) \vee A_{2}^{n}(y)}<0.5, \\
\frac{\breve{p}_{\min }^{p}}{A_{3}^{p}(x) \vee A_{3}^{p}(y)}<\frac{B_{3}^{p}(x, y)}{A_{3}^{p}(x) \vee A_{3}^{p}(y)}<0.5, & \frac{\breve{p}_{\min }^{n}}{A_{3}^{n}(x) \wedge A_{3}^{n}(y)}<\frac{B_{3}^{n}(x, y)}{A_{3}^{n}(x) \wedge A_{3}^{n}(y)}<0.5,
\end{array}
$$

therefore,

$B_{1}^{p}(x, y)=\left(A_{1}^{p}(x) \wedge A_{1}^{p}(y)\right) h_{1}\left(\mathcal{N}^{+}(x) \cap \mathcal{N}^{+}(y)\right)$, or $B_{1}^{p}(x, y)=\left[A_{1}^{p}(x) \wedge A_{1}^{p}(y)\right] \times m_{\max }^{p}$, or $\frac{B_{1}^{p}(x, y)}{\left(A_{1}^{p}(x) \wedge A_{1}^{p}(y)\right)}=m_{\max }^{p}$, or $\frac{B_{1}^{p}(x, y)}{\left(A_{1}^{p}(x) \wedge A_{1}^{p}(y)\right)^{2}}=\frac{m_{\max }^{p}}{\left(A_{1}^{p}(x) \wedge A_{1}^{p}(y)\right)}>0.5$,

$B_{2}^{p}(x, y)=\left(A_{2}^{p}(x) \wedge A_{2}^{p}(y)\right) h_{2}\left(\mathcal{N}^{+}(x) \cap \mathcal{N}^{+}(y)\right)$, or $B_{2}^{p}(x, y)=\left[A_{2}^{p}(x) \wedge A_{2}^{p}(y)\right] \times r_{\max }^{p}$,

or $\frac{B_{2}^{p}(x, y)}{\left(A_{2}^{p}(x) \wedge A_{2}^{p}(y)\right)}=r_{\max }^{p}$, or $\frac{B_{2}^{p}(x, y)}{\left(A_{2}^{p}(x) \wedge A_{2}^{p}(y)\right)^{2}}=\frac{r_{\max }^{p}}{\left(A_{2}^{p}(x) \wedge A_{2}^{p}(y)\right)}>0.5$,

$B_{3}^{p}(x, y)=\left(A_{3}^{p}(x) \vee A_{3}^{p}(y)\right) h_{3}\left(\mathcal{N}^{+}(x) \cap \mathcal{N}^{+}(y)\right)$, or $B_{3}^{p}(x, y)=\left[A_{3}^{p}(x) \vee A_{3}^{p}(y)\right] \times \breve{p}_{\min }^{p}$, or $\frac{B_{3}^{p}(x, y)}{\left(A_{3}^{p}(x) \vee A_{3}^{p}(y)\right)}=\breve{p}_{\min }^{p}$, or $\frac{B_{3}^{p}(x, y)}{\left(A_{3}^{p}(x) \vee A_{3}^{p}(y)\right)^{2}}=\frac{\breve{p}_{\min }^{p}}{\left(A_{3}^{p}(x) \vee A_{3}^{p}(y)\right)}<0.5$,

$B_{1}^{n}(x, y)=\left(A_{1}^{n}(x) \vee A_{1}^{n}(y)\right) h_{4}\left(\mathcal{N}^{+}(x) \cap \mathcal{N}^{+}(y)\right)$, or $B_{1}^{n}(x, y)=\left[A_{1}^{n}(x) \vee A_{1}^{n}(y)\right] \times m_{\max }^{n}$, or $\frac{B_{1}^{n}(x, y)}{\left(A_{1}^{n}(x) \vee A_{1}^{n}(y)\right)}=m_{\max }^{n}$, or $\frac{B_{1}^{n}(x, y)}{\left(A_{1}^{n}(x) \vee A_{1}^{n}(y)\right)^{2}}=\frac{m_{\max }^{n}}{\left(A_{1}^{n}(x) \vee A_{1}^{n}(y)\right)}<0.5$,

$B_{2}^{n}(x, y)=\left(A_{2}^{n}(x) \vee A_{2}^{n}(y)\right) h_{5}\left(\mathcal{N}^{+}(x) \cap \mathcal{N}^{+}(y)\right)$, or $B_{2}^{n}(x, y)=\left[A_{2}^{n}(x) \vee A_{2}^{n}(y)\right] \times r_{\max }^{n}$,

or $\frac{B_{2}^{n}(x, y)}{\left(A_{2}^{n}(x) \vee A_{2}^{n}(y)\right)}=r_{\max }^{n}$, or $\frac{B_{2}^{n}(x, y)}{\left(A_{2}^{n}(x) \vee A_{2}^{n}(y)\right)^{2}}=\frac{r_{\max }^{n}}{\left(A_{3}^{n}(x) \vee A_{2}^{n}(y)\right)}<0.5$, and

$B_{3}^{n}(x, y)=\left(A_{3}^{n}(x) \wedge A_{3}^{n}(y)\right) h_{6}\left(\mathcal{N}^{+}(x) \cap \mathcal{N}^{+}(y)\right)$, or $B_{3}^{n}(x, y)=\left[A_{3}^{n}(x) \wedge A_{3}^{n}(y)\right] \times \breve{p}_{\text {min }}^{n}$, or $\frac{B_{3}^{n}(x, y)}{\left(A_{3}^{n}(x) \wedge A_{3}^{n}(y)\right)}=\breve{p}_{\text {min }}^{n}$, or $\frac{B_{3}^{n}(x, y)}{\left(A_{3}^{n}(x) \wedge A_{3}^{n}(y)\right)^{2}}=\frac{\breve{p}_{\min }^{n}}{\left(A_{3}^{n}(x) \wedge A_{3}^{n}(y)\right)}<0.5$.

$$
\begin{aligned}
& \text { Hence, } \frac{B_{1}^{p}(x, y)}{\left(A_{1}^{p}(x) \wedge A_{1}^{p}(y)\right)^{2}}>0.5, \quad \frac{B_{1}^{n}(x, y)}{\left(A_{1}^{n}(x) \vee A_{1}^{n}(y)\right)^{2}}<0.5 \text {, } \\
& \frac{B_{2}^{p}(x, y)}{\left(A_{2}^{p}(x) \wedge A_{2}^{p}(y)\right)^{2}}>0.5, \quad \frac{B_{2}^{n}(x, y)}{\left(A_{2}^{n}(x) \vee A_{2}^{n}(y)\right)^{2}}<0.5, \\
& \frac{B_{3}^{p}(x, y)}{\left(A_{3}^{p}(x) \vee A_{3}^{p}(y)\right)^{2}}<0.5, \quad \frac{B_{3}^{n}(x, y)}{\left(A_{3}^{n}(x) \wedge A_{3}^{n}(y)\right)^{2}}<0.5,
\end{aligned}
$$

for all edges $(x, y)$ in $\mathcal{C}(\vec{G})$.

Definition 2.13. The bipolar single-valued neutrosophic open-neighborhood of a vertex $x$ of a bipolar single-valued neutrosophic graph $G=(A, B)$ is bipolar single-valued neutrosophic set $\mathcal{N}(x)=\left(X_{x}, t_{x}^{p}, i_{x}^{p}, f_{x}^{p}, t_{x}^{n}, i_{x}^{n}, f_{x}^{n}\right)$, where,

$$
X_{x}=\left\{y \mid B_{1}^{p}(x, y)>0, B_{2}^{p}(x, y)>0, B_{3}^{p}(x, y)>0, B_{1}^{n}(x, y)<0, B_{2}^{n}(x, y)<0, B_{3}^{n}(x, y)<0\right\}
$$

and $t_{x}^{p}: X_{x} \rightarrow[0,1]$ defined by $t_{x}^{p}(y)=B_{1}^{p}(x, y), i_{x}^{p}: X_{x} \rightarrow[0,1]$ defined by $i_{x}^{p}(y)=B_{2}^{p}(x$, $y), f_{x}^{p}: X_{x} \rightarrow[0,1]$ defined by $f_{x}^{p}(y)=B_{3}^{p}(x, y), t_{x}^{n}: X_{x} \rightarrow[-1,0]$ defined by $t_{x}^{n}(y)=$ $B_{1}^{n}(x, y), i_{x}^{n}: X_{x} \rightarrow[-1,0]$ defined by $i_{x}^{n}(y)=B_{2}^{n}(x, y)$, and $f_{x}^{n}: X_{x} \rightarrow[-1,0]$ defined by $f_{x}^{n}(y)=B_{3}^{n}(x, y)$. For every vertex $x \in X$, the bipolar single-valued neutrosophic singleton set, $\breve{A}_{x}=\left(x, A_{1}^{p \prime}, A_{2}^{p \prime}, A_{3}^{p \prime}, A_{1}^{n \prime}, A_{2}^{n \prime}, A_{3}^{n \prime}\right)$ such that: $A_{1}^{p^{\prime}}:\{x\} \rightarrow[0,1], A_{2}^{p \prime}:\{x\} \rightarrow[0,1]$, $A_{3}^{p \prime}:\{x\} \rightarrow[0,1], A_{1}^{n \prime}:\{x\} \rightarrow[-1,0], A_{2}^{n \prime}:\{x\} \rightarrow[-1,0], A_{3}^{n \prime}:\{x\} \rightarrow[-1,0]$, defined by $A_{1}^{p \prime}(x)=A_{1}^{p}(x), A_{2}^{p \prime}(x)=A_{2}^{p}(x), A_{3}^{p \prime}(x)=A_{3}^{p}(x), A_{1}^{n \prime}(x)=A_{1}^{n}(x), A_{2}^{n \prime}(x)=A_{2}^{n}(x)$, and 
$A_{3}^{n \prime}(x)=A_{3}^{n}(x)$, respectively. The bipolar single-valued neutrosophic closed-neighborhood of a vertex $x$ is $\mathcal{N}[x]=\mathcal{N}(x) \cup A_{x}$.

Definition 2.14. Suppose $G=(A, B)$ is a bipolar single-valued neutrosophic graph. Bipolar single-valued neutrosophic open-neighborhood graph of $G$ is a bipolar single-valued neutrosophic graph $\mathcal{N}(G)=\left(A, B^{\prime}\right)$ which has the same bipolar single-valued neutrosophic set of vertices in $G$ and has a bipolar single-valued neutrosophic edge between two vertices $x, y \in X$ in $\mathcal{N}(G)$ if and only if $\mathcal{N}(x) \cap \mathcal{N}(y)$ is a non-empty bipolar single-valued neutrosophic set in $G$. The positive truth-membership, indeterminacy-membership, falsity-membership and negative truthmembership, indeterminacy-membership, falsity-membership values of the edge $(x, y)$ are given by:

$B_{1}^{p \prime}(x, y)=\left[A_{1}^{p}(x) \wedge A_{1}^{p}(y)\right] h_{1}(\mathcal{N}(x) \cap \mathcal{N}(y)), \quad B_{1}^{n \prime}(x, y)=\left[A_{1}^{n}(x) \vee A_{1}^{n}(y)\right] h_{4}(\mathcal{N}(x) \cap \mathcal{N}(y))$,

$B_{2}^{p \prime}(x, y)=\left[A_{2}^{p}(x) \wedge A_{2}^{p}(y)\right] h_{2}(\mathcal{N}(x) \cap \mathcal{N}(y)), \quad B_{2}^{n \prime}(x, y)=\left[A_{2}^{n}(x) \vee A_{2}^{n}(y)\right] h_{5}(\mathcal{N}(x) \cap \mathcal{N}(y))$,

$B_{3}^{p^{\prime}}(x, y)=\left[A_{3}^{p}(x) \vee A_{3}^{p}(y)\right] h_{3}(\mathcal{N}(x) \cap \mathcal{N}(y)), \quad B_{3}^{n \prime}(x, y)=\left[A_{3}^{n}(x) \wedge A_{3}^{n}(y)\right] h_{6}(\mathcal{N}(x) \cap \mathcal{N}(y))$,

respectively.

Definition 2.15. Suppose $G=(A, B)$ is a bipolar single-valued neutrosophic graph. Bipolar single-valued neutrosophic closed-neighbourhood graph of $G$ is a single-valued bipolar singlevalued neutrosophic graph $\mathcal{N}(G)=\left(A, B^{\prime}\right)$ which has the same bipolar single-valued neutrosophic set of vertices in $G$ and has a bipolar single-valued neutrosophic edge between two vertices $x, y \in X$ in $\mathcal{N}[G]$ if and only if $\mathcal{N}[x] \cap \mathcal{N}[y]$ is a non-empty bipolar single-valued neutrosophic set in $G$. The positive truth-membership, indeterminacy-membership, falsity-membership and negative truth-membership, indeterminacy-membership, falsity-membership values of the edge $(x, y)$ are given by:

$$
\begin{array}{ll}
B_{1}^{p^{\prime}}(x, y)=\left[A_{1}^{p}(x) \wedge A_{1}^{p}(y)\right] h_{1}(\mathcal{N}[x] \cap \mathcal{N}[y]), & B_{1}^{n \prime}(x, y)=\left[A_{1}^{n}(x) \vee A_{1}^{n}(y)\right] h_{4}(\mathcal{N}[x] \cap \mathcal{N}[y]), \\
B_{2}^{p \prime}(x, y)=\left[A_{2}^{p}(x) \wedge A_{2}^{p}(y)\right] h_{2}(\mathcal{N}[x] \cap \mathcal{N}[y]), & B_{2}^{n \prime}(x, y)=\left[A_{2}^{n}(x) \vee A_{2}^{n}(y)\right] h_{5}(\mathcal{N}[x] \cap \mathcal{N}[y]), \\
B_{3}^{p \prime}(x, y)=\left[A_{3}^{p}(x) \vee A_{3}^{p}(y)\right] h_{3}(\mathcal{N}[x] \cap \mathcal{N}[y]), & B_{3}^{n \prime}(x, y)=\left[A_{3}^{n}(x) \wedge A_{3}^{n}(y)\right] h_{6}(\mathcal{N}[x] \cap \mathcal{N}[y]),
\end{array}
$$

respectively.

Example 2.5. Consider $G=(A, B)$ is a bipolar single-valued neutrosophic digraph, such that, $X=\{a, b, c, d\}, A=\{(a, 0.7,0.8,0.6,-0.5,-0.3,-0.7),(b, 0.3,0.5,0.7,-0.7,-0.8,-0.9),(c$, $0.8,0.9,0.7,-0.5,-0.6,-0.7),(d, 0.8,0.7,0.6,-0.3,-0.7,-0.8)\}$, and $B=\{((a, b), 0.2,0.4,0.6$, $-0.4,-0.2,-0.8),((a, c), 0.6,0.7,0.6,-0.5,-0.2,-0.6),((b, d), 0.2,0.4,0.6,-0.2,-0.6,-0.8),((c, d)$, $0.6,0.6,0.5,-0.2,-0.5,-0.7)\}$, as shown in Fig.5.

Then corresponding bipolar single-valued neutrosophic open and closed-neighbourhood graphs are shown in Figs. 6 and 7, respectively.

Theorem 2.3. For each edge of a bipolar single-valued neutrosophic graph $G$, there exists an edge in $\mathcal{N}[G]$.

Proof. Suppose $(x, y)$ is an edge of a bipolar single-valued neutrosophic graph $G=(A, B)$. Suppose $\mathcal{N}[G]=\left(A, B^{\prime}\right)$ is the corresponding closed-neighborhood of a bipolar single-valued 


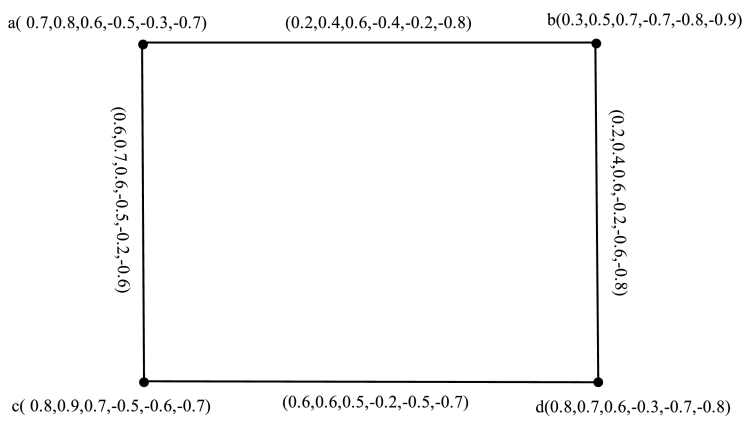

Figure 5: Bipolar single-valued neutrosophic graph

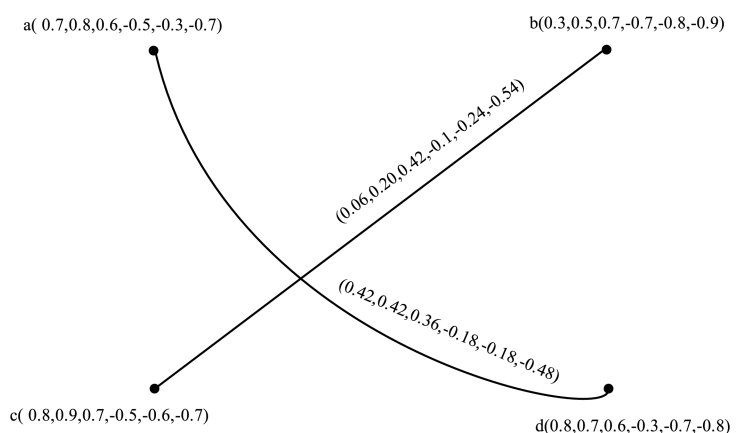

Figure 6: $\mathcal{N}(G)$

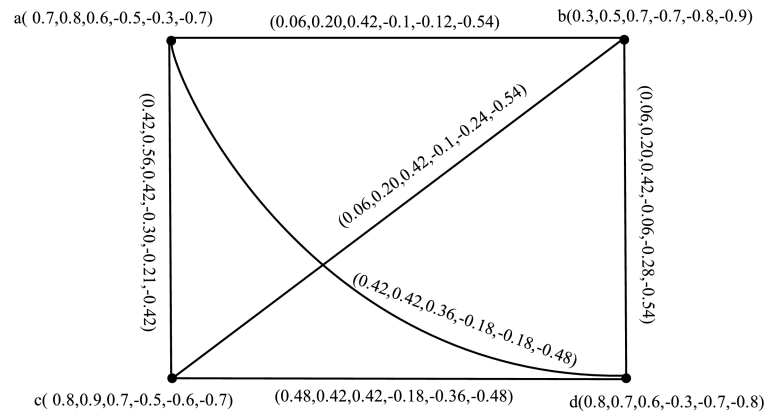

Figure 7: $\mathcal{N}[G]$

neutrosophic graph. Suppose $x, y \in \mathcal{N}[x]$ and $x, y \in \mathcal{N}[y]$. Then $x, y \in \mathcal{N}[x] \cap \mathcal{N}[y]$. Hence,

$$
\begin{array}{ll}
h_{1}(\mathcal{N}[x] \cap \mathcal{N}[y]) \neq 0, & h_{4}(\mathcal{N}[x] \cap \mathcal{N}[y]) \neq 0, \\
h_{2}(\mathcal{N}[x] \cap \mathcal{N}[y]) \neq 0, & h_{5}(\mathcal{N}[x] \cap \mathcal{N}[y]) \neq 0, \\
h_{3}(\mathcal{N}[x] \cap \mathcal{N}[y]) \neq 0, & h_{6}(\mathcal{N}[x] \cap \mathcal{N}[y]) \neq 0 .
\end{array}
$$


Then,

$$
\begin{aligned}
& B_{1}^{p \prime}(x, y)=\left[A_{1}^{p}(x) \wedge A_{1}^{p}(y)\right] h_{1}(\mathcal{N}[x] \cap \mathcal{N}[y]) \neq 0, \\
& B_{1}^{n \prime}(x, y)=\left[A_{1}^{n}(x) \vee A_{1}^{n}(y)\right] h_{4}(\mathcal{N}[x] \cap \mathcal{N}[y]) \neq 0, \\
& B_{2}^{p \prime}(x, y)=\left[A_{2}^{p}(x) \wedge A_{2}^{p}(y)\right] h_{2}(\mathcal{N}[x] \cap \mathcal{N}[y]) \neq 0, \\
& B_{2}^{n \prime}(x, y)=\left[A_{2}^{n}(x) \vee A_{2}^{n}(y)\right] h_{5}(\mathcal{N}[x] \cap \mathcal{N}[y]) \neq 0, \\
& B_{3}^{p \prime}(x, y)=\left[A_{3}^{p}(x) \vee A_{3}^{p}(y)\right] h_{3}(\mathcal{N}[x] \cap \mathcal{N}[y]) \neq 0, \\
& B_{3}^{n \prime}(x, y)=\left[A_{3}^{n}(x) \wedge A_{3}^{n}(y)\right] h_{6}(\mathcal{N}[x] \cap \mathcal{N}[y]) \neq 0 .
\end{aligned}
$$

Thus, for each edge $(x, y)$ in bipolar single-valued neutrosophic graph $G$, there exists an edge $(x, y)$ in $\mathcal{N}[G]$.

We now discuss the method of construction of bipolar single-valued neutrosophic competition graph of the Cartesian product of bipolar single-valued neutrosophic digraph in following theorem.

Theorem 2.4. em Let $\mathcal{C}\left(\overrightarrow{G_{1}}\right)=\left(A_{1}, B_{1}\right)$ and $\mathcal{C}\left(\overrightarrow{G_{2}}\right)=\left(A_{2}, B_{2}\right)$ be two bipolar single-valued neutrosophic competition graphs of bipolar single-valued neutrosophic digraphs $\overrightarrow{G_{1}}=\left(A_{1}, \overrightarrow{l_{1}}\right)$ and $\overrightarrow{G_{2}}=\left(A_{2}, \overrightarrow{l_{2}}\right)$, respectively. Then $\mathcal{C}\left(\overrightarrow{G_{1}} \square \overrightarrow{G_{2}}\right)=G_{\mathcal{C}\left(\overrightarrow{G_{1}}\right) * \square \mathcal{C}\left(\overrightarrow{G_{2}}\right)^{*}} \cup G^{\square}$ where, $G_{\mathcal{C}\left(\overrightarrow{G_{1}}\right)^{*} \square \mathcal{C}\left(\overrightarrow{G_{2}}\right)^{*}}$ is a bipolar single-valued neutrosophic graph on the crisp graph $\left(X_{1} \times X_{2}, E_{\mathcal{C}\left(\overrightarrow{G_{1}}\right)^{*}} \square E_{\mathcal{C}\left(\overrightarrow{G_{2}}\right)^{*}}\right)$, $\mathcal{C}\left(\overrightarrow{G_{1}}\right)^{*}$ and $\mathcal{C}\left(\overrightarrow{G_{2}}\right)^{*}$ are the crisp competition graphs of $\overrightarrow{G_{1}}$ and $\overrightarrow{G_{2}}$, respectively. $G^{\square}$ is a bipolar single-valued neutrosophic graph on $\left(X_{1} \times X_{2}, E^{\square}\right)$ such that:

1. $E^{\square}=\left\{\left(x_{1}, x_{2}\right)\left(y_{1}, y_{2}\right): y_{1} \in \mathcal{N}^{-}\left(x_{1}\right)^{*}, y_{2} \in \mathcal{N}^{+}\left(x_{2}\right)^{*}\right\}$

$E_{\mathcal{C}\left(\overrightarrow{G_{1}}\right)^{*}} \square E_{\mathcal{C}\left(\overrightarrow{G_{2}}\right)^{*}}=\left\{\left(x_{1}, x_{2}\right)\left(x_{1}, y_{2}\right): x_{1} \in X_{1}, x_{2} y_{2} \in E_{\mathcal{C}\left(\overrightarrow{G_{2}}\right)^{*}}\right\} \cup\left\{\left(x_{1}, x_{2}\right)\left(y_{1}, x_{2}\right): x_{2} \in\right.$ $\left.X_{2}, x_{1} y_{1} \in E_{\mathcal{C}\left(\overrightarrow{G_{1}}\right)^{*}}\right\}$.

2. $t_{A_{1} \square A_{2}}^{p}=t_{A_{1}}^{p}\left(x_{1}\right) \wedge t_{A_{2}}^{p}\left(x_{2}\right), \quad i_{A_{1} \square A_{2}}^{p}=i_{A_{1}}^{p}\left(x_{1}\right) \wedge i_{A_{2}}^{p}\left(x_{2}\right), \quad f_{A_{1} \square A_{2}}^{p}=f_{A_{1}}^{p}\left(x_{1}\right) \vee f_{A_{2}}^{p}\left(x_{2}\right)$, $t_{A_{1} \square A_{2}}^{n}=t_{A_{1}}^{n}\left(x_{1}\right) \vee t_{A_{2}}^{n}\left(x_{2}\right), \quad i_{A_{1} \square A_{2}}^{n}=i_{A_{1}}^{n}\left(x_{1}\right) \vee i_{A_{2}}^{n}\left(x_{2}\right), \quad f_{A_{1} \square A_{2}}^{n}=f_{A_{1}}^{n}\left(x_{1}\right) \wedge f_{A_{2}}^{n}\left(x_{2}\right)$.

3. $t_{B}^{p}\left(\left(x_{1}, x_{2}\right)\left(x_{1}, y_{2}\right)\right)=\left[t_{A_{1}}^{p}\left(x_{1}\right) \wedge t_{A_{2}}^{p}\left(x_{2}\right) \wedge t_{A_{2}}^{p}\left(y_{2}\right)\right] \times \vee_{a_{2}}\left\{t_{A_{1}}^{p}\left(x_{1}\right) \wedge t_{\overrightarrow{l_{2}}}^{p}\left(x_{2} a_{2}\right) \wedge t_{\overrightarrow{l_{2}}}^{p}\left(y_{2} a_{2}\right)\right\}$, $\left(x_{1}, x_{2}\right)\left(x_{1}, y_{2}\right) \in E_{\mathcal{C}\left(\overrightarrow{G_{1}}\right)^{*}} \square E_{\mathcal{C}\left(\overrightarrow{G_{2}}\right)^{*}}, \quad a_{2} \in\left(\mathcal{N}^{+}\left(x_{2}\right) \cap \mathcal{N}^{+}\left(y_{2}\right)\right)^{*}$.

4. $i_{B}^{p}\left(\left(x_{1}, x_{2}\right)\left(x_{1}, y_{2}\right)\right)=\left[i_{A_{1}}^{p}\left(x_{1}\right) \wedge i_{A_{2}}^{p}\left(x_{2}\right) \wedge i_{A_{2}}^{p}\left(y_{2}\right)\right] \times \vee_{a_{2}}\left\{i_{A_{1}}^{p}\left(x_{1}\right) \wedge i_{\overrightarrow{l_{2}}}^{p}\left(x_{2} a_{2}\right) \wedge i_{\vec{l}_{2}}^{p}\left(y_{2} a_{2}\right)\right\}$, $\left(x_{1}, x_{2}\right)\left(x_{1}, y_{2}\right) \in E_{\mathcal{C}\left(\overrightarrow{G_{1}}\right)^{*}} \square E_{\mathcal{C}\left(\overrightarrow{G_{2}}\right)^{*}}, \quad a_{2} \in\left(\mathcal{N}^{+}\left(x_{2}\right) \cap \mathcal{N}^{+}\left(y_{2}\right)\right)^{*}$.

5. $f_{B}^{p}\left(\left(x_{1}, x_{2}\right)\left(x_{1}, y_{2}\right)\right)=\left[f_{A_{1}}^{p}\left(x_{1}\right) \vee f_{A_{2}}^{p}\left(x_{2}\right) \vee f_{A_{2}}^{p}\left(y_{2}\right)\right] \times \vee_{a_{2}}\left\{f_{A_{1}}^{p}\left(x_{1}\right) \vee f_{\overrightarrow{l_{2}}}^{p}\left(x_{2} a_{2}\right) \vee f_{\overrightarrow{l_{2}}}^{p}\left(y_{2} a_{2}\right)\right\}$, $\left(x_{1}, x_{2}\right)\left(x_{1}, y_{2}\right) \in E_{\mathcal{C}\left(\overrightarrow{G_{1}}\right)^{*}} \square E_{\mathcal{C}\left(\overrightarrow{G_{2}}\right)^{*}}, \quad a_{2} \in\left(\mathcal{N}^{+}\left(x_{2}\right) \cap \mathcal{N}^{+}\left(y_{2}\right)\right)^{*}$.

6. $t_{B}^{n}\left(\left(x_{1}, x_{2}\right)\left(x_{1}, y_{2}\right)\right)=\left[t_{A_{1}}^{n}\left(x_{1}\right) \vee t_{A_{2}}^{n}\left(x_{2}\right) \vee t_{A_{2}}^{n}\left(y_{2}\right)\right] \times \vee_{a_{2}}\left\{t_{A_{1}}^{n}\left(x_{1}\right) \vee t_{\overrightarrow{l_{2}}}^{n}\left(x_{2} a_{2}\right) \vee t_{\overrightarrow{l_{2}}}^{n}\left(y_{2} a_{2}\right)\right\}$, $\left(x_{1}, x_{2}\right)\left(x_{1}, y_{2}\right) \in E_{\mathcal{C}\left(\overrightarrow{G_{1}}\right)^{*}} \square E_{\mathcal{C}\left(\overrightarrow{G_{2}}\right)^{*}}, \quad a_{2} \in\left(\mathcal{N}^{+}\left(x_{2}\right) \cap \mathcal{N}^{+}\left(y_{2}\right)\right)^{*}$.

7. $i_{B}^{n}\left(\left(x_{1}, x_{2}\right)\left(x_{1}, y_{2}\right)\right)=\left[i_{A_{1}}^{n}\left(x_{1}\right) \vee i_{A_{2}}^{n}\left(x_{2}\right) \vee i_{A_{2}}^{n}\left(y_{2}\right)\right] \times \vee_{a_{2}}\left\{i_{A_{1}}^{n}\left(x_{1}\right) \vee i \frac{\vec{l}_{2}}{l_{2}}\left(x_{2} a_{2}\right) \vee i_{l_{2}}^{n}\left(y_{2} a_{2}\right)\right\}$, $\left(x_{1}, x_{2}\right)\left(x_{1}, y_{2}\right) \in E_{\mathcal{C}\left(\overrightarrow{G_{1}}\right)^{*}} \square E_{\mathcal{C}\left(\overrightarrow{G_{2}}\right)^{*}}, \quad a_{2} \in\left(\mathcal{N}^{+}\left(x_{2}\right) \cap \mathcal{N}^{+}\left(y_{2}\right)\right)^{*}$. 
8. $f_{B}^{n}\left(\left(x_{1}, x_{2}\right)\left(x_{1}, y_{2}\right)\right)=\left[f_{A_{1}}^{n}\left(x_{1}\right) \wedge f_{A_{2}}^{n}\left(x_{2}\right) \wedge f_{A_{2}}^{n}\left(y_{2}\right)\right] \times \vee_{a_{2}}\left\{f_{A_{1}}^{n}\left(x_{1}\right) \wedge f_{l_{2}}^{n}\left(x_{2} a_{2}\right) \wedge f_{\overrightarrow{l_{2}}}^{n}\left(y_{2} a_{2}\right)\right\}$, $\left(x_{1}, x_{2}\right)\left(x_{1}, y_{2}\right) \in E_{\mathcal{C}\left(\overrightarrow{G_{1}}\right)^{*}} \square E_{\mathcal{C}\left(\overrightarrow{G_{2}}\right)^{*}}, \quad a_{2} \in\left(\mathcal{N}^{+}\left(x_{2}\right) \cap \mathcal{N}^{+}\left(y_{2}\right)\right)^{*}$.

9. $t_{B}^{p}\left(\left(x_{1}, x_{2}\right)\left(y_{1}, x_{2}\right)\right)=\left[t_{A_{1}}^{p}\left(x_{1}\right) \wedge t_{A_{1}}^{p}\left(y_{1}\right) \wedge t_{A_{2}}^{p}\left(x_{2}\right)\right] \times \vee_{a_{1}}\left\{t_{A_{2}}^{p}\left(x_{2}\right) \wedge t_{\overrightarrow{l_{1}}}^{p}\left(x_{1} a_{1}\right) \wedge t_{\overrightarrow{l_{1}}}^{p}\left(y_{1} a_{1}\right)\right\}$, $\left(x_{1}, x_{2}\right)\left(y_{1}, x_{2}\right) \in E_{\mathcal{C}\left(\overrightarrow{G_{1}}\right)^{*}} \square E_{\mathcal{C}\left(\overrightarrow{G_{2}}\right)^{*}}, \quad a_{1} \in\left(\mathcal{N}^{+}\left(x_{1}\right) \cap \mathcal{N}^{+}\left(y_{1}\right)\right)^{*}$.

10. $i_{B}^{p}\left(\left(x_{1}, x_{2}\right)\left(y_{1}, x_{2}\right)\right)=\left[i_{A_{1}}^{p}\left(x_{1}\right) \wedge i_{A_{1}}^{p}\left(y_{1}\right) \wedge i_{A_{2}}^{p}\left(x_{2}\right)\right] \times \vee_{a_{1}}\left\{i_{A_{2}}^{p}\left(x_{2}\right) \wedge i_{l_{1}}^{p}\left(x_{1} a_{1}\right) \wedge i_{\overrightarrow{l_{1}}}^{p}\left(y_{1} a_{1}\right)\right\}$, $\left(x_{1}, x_{2}\right)\left(y_{1}, x_{2}\right) \in E_{\mathcal{C}\left(\overrightarrow{G_{1}}\right)^{*}} \square E_{\mathcal{C}\left(\overrightarrow{G_{2}}\right)^{*}}, \quad a_{1} \in\left(\mathcal{N}^{+}\left(x_{1}\right) \cap \mathcal{N}^{+}\left(y_{1}\right)\right)^{*}$.

11. $f_{B}^{p}\left(\left(x_{1}, x_{2}\right)\left(y_{1}, x_{2}\right)\right)=\left[f_{A_{1}}^{p}\left(x_{1}\right) \vee f_{A_{1}}^{p}\left(y_{1}\right) \vee f_{A_{2}}^{p}\left(x_{2}\right)\right] \times \vee_{a_{1}}\left\{f_{A_{2}}^{p}\left(x_{2}\right) \vee f_{\overrightarrow{l_{1}}}^{p}\left(x_{1} a_{1}\right) \vee f_{\overrightarrow{l_{1}}}^{p}\left(y_{1} a_{1}\right)\right\}$, $\left(x_{1}, x_{2}\right)\left(y_{1}, x_{2}\right) \in E_{\mathcal{C}\left(\overrightarrow{G_{1}}\right)^{*}} \square E_{\mathcal{C}\left(\overrightarrow{G_{2}}\right)^{*}}, \quad a_{1} \in\left(\mathcal{N}^{+}\left(x_{1}\right) \cap \mathcal{N}^{+}\left(y_{1}\right)\right)^{*}$.

12. $t_{B}^{n}\left(\left(x_{1}, x_{2}\right)\left(y_{1}, x_{2}\right)\right)=\left[t_{A_{1}}^{n}\left(x_{1}\right) \vee t_{A_{1}}^{n}\left(y_{1}\right) \vee t_{A_{2}}^{n}\left(x_{2}\right)\right] \times \vee_{a_{1}}\left\{t_{A_{2}}^{n}\left(x_{2}\right) \vee t_{l_{1}}^{n}\left(x_{1} a_{1}\right) \vee t_{\vec{l}_{1}}^{n}\left(y_{1} a_{1}\right)\right\}$, $\left(x_{1}, x_{2}\right)\left(y_{1}, x_{2}\right) \in E_{\mathcal{C}\left(\overrightarrow{G_{1}}\right)^{*}} \square E_{\mathcal{C}\left(\overrightarrow{G_{2}}\right)^{*}}, \quad a_{1} \in\left(\mathcal{N}^{+}\left(x_{1}\right) \cap \mathcal{N}^{+}\left(y_{1}\right)\right)^{*}$.

13. $i_{B}^{n}\left(\left(x_{1}, x_{2}\right)\left(y_{1}, x_{2}\right)\right)=\left[i_{A_{1}}^{n}\left(x_{1}\right) \vee i_{A_{1}}^{n}\left(y_{1}\right) \vee i_{A_{2}}^{n}\left(x_{2}\right)\right] \times \vee_{a_{1}}\left\{i_{A_{2}}^{n}\left(x_{2}\right) \vee i_{\overrightarrow{l_{1}}}^{n}\left(x_{1} a_{1}\right) \vee i_{\overrightarrow{l_{1}}}^{n}\left(y_{1} a_{1}\right)\right\}$, $\left(x_{1}, x_{2}\right)\left(y_{1}, x_{2}\right) \in E_{\mathcal{C}\left(\overrightarrow{G_{1}}\right)^{*}} \square E_{\mathcal{C}\left(\overrightarrow{G_{2}}\right)^{*}}, \quad a_{1} \in\left(\mathcal{N}^{+}\left(x_{1}\right) \cap \mathcal{N}^{+}\left(y_{1}\right)\right)^{*}$.

14. $f_{B}^{n}\left(\left(x_{1}, x_{2}\right)\left(y_{1}, x_{2}\right)\right)=\left[f_{A_{1}}^{n}\left(x_{1}\right) \wedge f_{A_{1}}^{n}\left(y_{1}\right) \wedge f_{A_{2}}^{n}\left(x_{2}\right)\right] \times \vee_{a_{1}}\left\{f_{A_{2}}^{n}\left(x_{2}\right) \wedge f \frac{n}{\vec{l}_{1}}\left(x_{1} a_{1}\right) \wedge f_{\overrightarrow{l_{1}}}^{n}\left(y_{1} a_{1}\right)\right\}$, $\left(x_{1}, x_{2}\right)\left(y_{1}, x_{2}\right) \in E_{\mathcal{C}\left(\overrightarrow{G_{1}}\right)^{*}} \square E_{\mathcal{C}\left(\overrightarrow{G_{2}}\right)^{*}}, \quad a_{1} \in\left(\mathcal{N}^{+}\left(x_{1}\right) \cap \mathcal{N}^{+}\left(y_{1}\right)\right)^{*}$.

15. $t_{B}^{p}\left(\left(x_{1}, x_{2}\right)\left(y_{1}, y_{2}\right)\right)=\left[t_{A_{1}}^{p}\left(x_{1}\right) \wedge t_{A_{1}}^{p}\left(y_{1}\right) \wedge t_{A_{2}}^{p}\left(x_{2}\right) \wedge t_{A_{2}}^{p}\left(y_{2}\right)\right] \times\left[t_{A_{1}}^{p}\left(x_{1}\right) \wedge t_{\overrightarrow{l_{1}}}^{p}\left(y_{1} x_{1}\right) \wedge t_{A_{2}}^{p}\left(y_{2}\right) \wedge\right.$ $\left.t_{\overrightarrow{l_{2}}}^{p}\left(x_{2} y_{2}\right)\right], \quad\left(x_{1}, y_{1}\right)\left(x_{2}, y_{2}\right) \in E^{\square}$.

16. $i_{B}^{p}\left(\left(x_{1}, x_{2}\right)\left(y_{1}, y_{2}\right)\right)=\left[i_{A_{1}}^{p}\left(x_{1}\right) \wedge i_{A_{1}}^{p}\left(y_{1}\right) \wedge i_{A_{2}}^{p}\left(x_{2}\right) \wedge i_{A_{2}}^{p}\left(y_{2}\right)\right] \times\left[i_{A_{1}}^{p}\left(x_{1}\right) \wedge i_{\overrightarrow{l_{1}}}^{p}\left(y_{1} x_{1}\right) \wedge i_{A_{2}}^{p}\left(y_{2}\right) \wedge\right.$ $\left.i_{\overrightarrow{l_{2}}}^{p}\left(x_{2} y_{2}\right)\right], \quad\left(x_{1}, y_{1}\right)\left(x_{2}, y_{2}\right) \in E^{\square}$.

17. $f_{B}^{p}\left(\left(x_{1}, x_{2}\right)\left(y_{1}, y_{2}\right)\right)=\left[f_{A_{1}}^{p}\left(x_{1}\right) \vee f_{A_{1}}^{p}\left(y_{1}\right) \vee f_{A_{2}}^{p}\left(x_{2}\right) \vee f_{A_{2}}^{p}\left(y_{2}\right)\right] \times\left[f_{A_{1}}^{p}\left(x_{1}\right) \vee f_{\overrightarrow{l_{1}}}^{p}\left(y_{1} x_{1}\right) \vee\right.$ $\left.f_{A_{2}}^{p}\left(y_{2}\right) \vee f_{\overrightarrow{l_{2}}}^{p}\left(x_{2} y_{2}\right)\right], \quad\left(x_{1}, y_{1}\right)\left(x_{2}, y_{2}\right) \in E^{\square}$.

18. $t_{B}^{n}\left(\left(x_{1}, x_{2}\right)\left(y_{1}, y_{2}\right)\right)=\left[t_{A_{1}}^{n}\left(x_{1}\right) \vee t_{A_{1}}^{n}\left(y_{1}\right) \vee t_{A_{2}}^{n}\left(x_{2}\right) \vee t_{A_{2}}^{n}\left(y_{2}\right)\right] \times\left[t_{A_{1}}^{n}\left(x_{1}\right) \vee t_{\overrightarrow{l_{1}}}^{n}\left(y_{1} x_{1}\right) \vee t_{A_{2}}^{n}\left(y_{2}\right) \vee\right.$ $\left.t_{\overrightarrow{l_{2}}}^{n}\left(x_{2} y_{2}\right)\right], \quad\left(x_{1}, y_{1}\right)\left(x_{2}, y_{2}\right) \in E^{\square}$.

19. $i_{B}^{n}\left(\left(x_{1}, x_{2}\right)\left(y_{1}, y_{2}\right)\right)=\left[i_{A_{1}}^{n}\left(x_{1}\right) \vee i_{A_{1}}^{n}\left(y_{1}\right) \vee i_{A_{2}}^{n}\left(x_{2}\right) \vee i_{A_{2}}^{n}\left(y_{2}\right)\right] \times\left[i_{A_{1}}^{n}\left(x_{1}\right) \vee i_{\overrightarrow{l_{1}}}^{n}\left(y_{1} x_{1}\right) \vee i_{A_{2}}^{n}\left(y_{2}\right) \vee\right.$ $\left.i \vec{l}_{l_{2}}^{n}\left(x_{2} y_{2}\right)\right], \quad\left(x_{1}, y_{1}\right)\left(x_{2}, y_{2}\right) \in E^{\square}$.

20. $f_{B}^{n}\left(\left(x_{1}, x_{2}\right)\left(y_{1}, y_{2}\right)\right)=\left[f_{A_{1}}^{n}\left(x_{1}\right) \wedge f_{A_{1}}^{n}\left(y_{1}\right) \wedge f_{A_{2}}^{n}\left(x_{2}\right) \wedge f_{A_{2}}^{n}\left(y_{2}\right)\right] \times\left[f_{A_{1}}^{n}\left(x_{1}\right) \wedge f_{\overrightarrow{l_{1}}}^{n}\left(y_{1} x_{1}\right) \wedge\right.$ $\left.f_{A_{2}}^{n}\left(y_{2}\right) \wedge f \frac{n}{l_{2}}\left(x_{2} y_{2}\right)\right], \quad\left(x_{1}, y_{1}\right)\left(x_{2}, y_{2}\right) \in E^{\square}$.

Proof. Using similar arguments as in Theorem 2.3[14], it can be proved.

Example 2.6. Consider $\overrightarrow{G_{1}}=\left(A_{1}, l_{1}\right)$ and $\overrightarrow{G_{2}}=\left(A_{2}, l_{2}\right)$ be two bipolar single-valued neutrosophic digraphs, respectively as shown in Fig.8. The bipolar single-valued neutrosophic out and in-neighborhoods of $\overrightarrow{G_{1}}$ and $\overrightarrow{G_{2}}$ are given in Tables 5 and 6 .

The bipolar single-valued neutrosophic competition graphs $\mathcal{C}\left(\vec{G}_{1}\right)$ and $\mathcal{C}\left(\vec{G}_{2}\right)$ are given in Fig.9. 


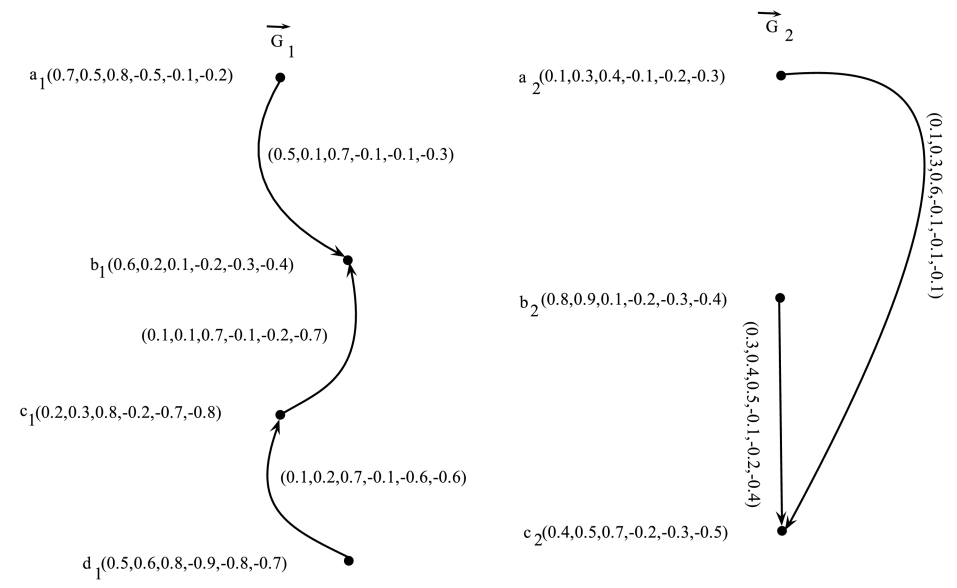

Figure 8: Bipolar single-valued neutrosophic digraphs

Table 5: Bipolar single-valued neutrosophic-out and in-neighborhoods of $\vec{G}_{1}$

\begin{tabular}{|l|l|l|}
\hline$x \in X_{1}$ & $\mathcal{N}^{+}(x)$ & $\mathcal{N}^{-}(x)$ \\
\hline$a_{1}$ & $\left\{b_{1}(0.5,0.1,0.7,-0.1,-0.1,-0.3)\right\}$ & $\emptyset$ \\
$b_{1}$ & $\emptyset$ & $\left\{a_{1}(0.5,0.1,0.7,-0.1,-0.1,-0.3)\right\}$ \\
$c_{1}$ & $\left\{b_{1}(0.1,0.1,0.7,-0.1,-0.2,-0.7)\right\}$ & $\left\{d_{1}(0.1,0.2,0.7,-0.1,-0.6,-0.6)\right\}$ \\
$d_{1}$ & $\left\{c_{1}(0.1,0.2,0.7,-0.1,-0.6,-0.6)\right\}$ & $\emptyset$ \\
\hline
\end{tabular}

Table 6: Bipolar single-valued neutrosophic-out and in-neighborhoods of $\vec{G}_{2}$

\begin{tabular}{|l|l|l|}
\hline$x \in X_{2}$ & $\mathcal{N}^{+}(x)$ & $\mathcal{N}^{-}(x)$ \\
\hline$a_{2}$ & $\left\{c_{2}(0.1,0.3,0.6,-0.1,-0.1,-0.1)\right\}$ & $\emptyset$ \\
$b_{2}$ & $\left\{c_{2}(0.3,0.4,0.5,-0.1,-0.2,-0.4)\right\}$ & $\emptyset$ \\
$c_{2}$ & $\emptyset$ & $\left\{a_{2}(0.1,0.3,0.6,-0.1,-0.1,-0.1), b_{2}(0.3,0.4,0.5,-0.1,-0.2,-0.4)\right\}$ \\
\hline
\end{tabular}

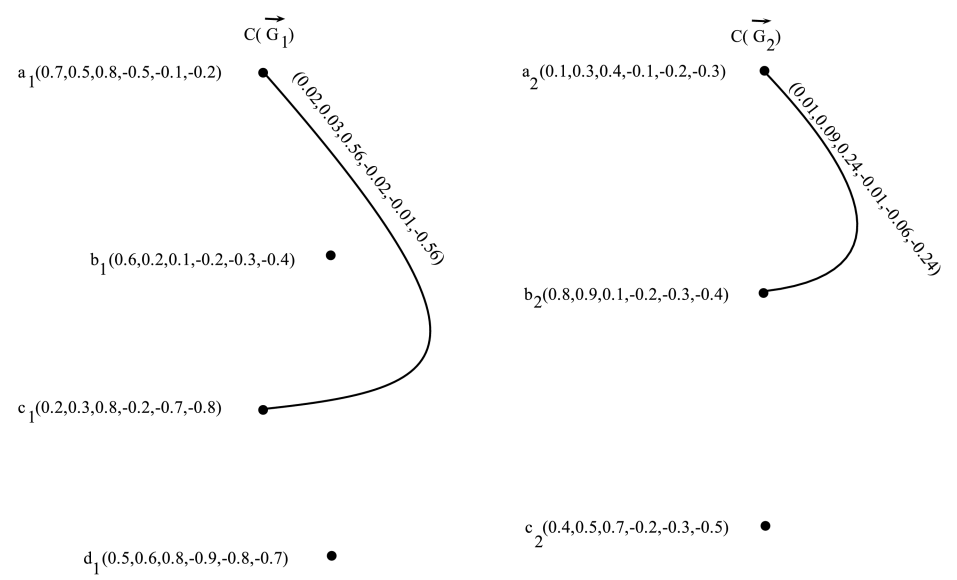

Figure 9: Bipolar single-valued neutrosophic competition graphs of $\overrightarrow{G_{1}}$ and $\overrightarrow{G_{2}}$ 
We now construct the bipolar single-valued neutrosophic competition graph $G_{\mathcal{C}\left(\overrightarrow{G_{1}}\right) * \square \mathcal{C}\left(\overrightarrow{G_{2}}\right) *} \cup$ $G^{\square}=(W, B)$, where, $W=\left(t_{W}^{p}, i_{W}^{p}, f_{W}^{p}, t_{W}^{n}, i_{W}^{n}, f_{W}^{n}\right)$ and $B=\left(t_{B}^{p}, i_{B}^{p}, f_{B}^{p}, t_{B}^{n}, i_{B}^{n}, f_{B}^{n}\right)$, from $\mathcal{C}\left(\overrightarrow{G_{1}}\right)^{*}$ and $\mathcal{C}\left(\overrightarrow{G_{2}}\right)^{*}$ using Theorem 1.4. We obtained the following sets of edges by using condition (1).

$$
\begin{aligned}
E_{\mathcal{C}\left(\overrightarrow{G_{1}}\right) * \square \mathcal{C}\left(\overrightarrow{G_{2}}\right)^{*}=} & \left\{\left(a_{1}, a_{2}\right)\left(a_{1}, b_{2}\right),\left(b_{1}, a_{2}\right)\left(b_{1}, b_{2}\right),\left(c_{1}, a_{2}\right)\left(c_{1}, b_{2}\right),\right. \\
& \left(d_{1}, a_{2}\right)\left(d_{1}, b_{2}\right),\left(a_{1}, a_{2}\right)\left(c_{1}, a_{2}\right), \\
& \left.\left(a_{1}, b_{2}\right)\left(c_{1}, b_{2}\right),\left(a_{1}, c_{2}\right)\left(c_{1}, c_{2}\right)\right\}, \\
E^{\square}= & \left\{\left(b_{1}, a_{2}\right)\left(a_{1}, c_{2}\right),\left(b_{1}, a_{2}\right)\left(c_{1}, c_{2}\right),\left(b_{1}, b_{2}\right)\left(a_{1}, c_{2}\right),\right. \\
& \left.\left(b_{1}, b_{2}\right)\left(c_{1}, c_{2}\right),\left(c_{1}, a_{2}\right)\left(d_{1}, c_{2}\right),\left(c_{1}, b_{2}\right)\left(d_{1}, c_{2}\right)\right\} .
\end{aligned}
$$

According to conditions (3) to (20), the degrees of positive truth-membership, indeterminacymembership, falsity-membership, negative truth-membership, indeterminacy-membership and

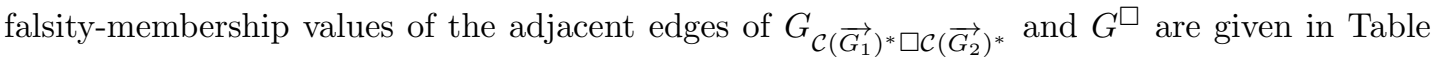
7 .

Table 7: Adjacent edges of $G_{\mathcal{C}\left(\overrightarrow{G_{1}}\right) * \square \mathcal{C}\left(\overrightarrow{G_{2}}\right)^{*}} \cup G^{\square}$

\begin{tabular}{|l|l|}
\hline$\left(x_{1}, x_{2}\right)\left(y_{1}, y_{2}\right)$ & $B\left(x_{1}, x_{2}\right)\left(y_{1}, y_{2}\right)$ \\
\hline$\left(a_{1}, a_{2}\right)\left(a_{1}, b_{2}\right)$ & $(0.01,0.09,0.64,-0.01,-0.03,-0.32)$ \\
$\left(b_{1}, a_{2}\right)\left(b_{1}, b_{2}\right)$ & $(0.01,0.04,0.24,-0.01,-0.04,-0.24)$ \\
$\left(c_{1}, a_{2}\right)\left(c_{1}, b_{2}\right)$ & $(0.01,0.09,0.64,-0.01,-0.06,-0.64)$ \\
$\left(d_{1}, a_{2}\right)\left(d_{1}, b_{2}\right)$ & $(0.01,0.09,0.64,-0.01,-0.06,-0.56)$ \\
$\left(a_{1}, a_{2}\right)\left(c_{1}, a_{2}\right)$ & $(0.01,0.03,0.56,-0.01,-0.01,-0.56)$ \\
$\left(a_{1}, b_{2}\right)\left(c_{1}, b_{2}\right)$ & $(0.02,0.03,0.56,-0.02,-0.01,-0.56)$ \\
$\left(a_{1}, c_{2}\right)\left(c_{1}, c_{2}\right)$ & $(0.02,0.03,0.56,-0.02,-0.01,-0.56)$ \\
$\left(b_{1}, a_{2}\right)\left(a_{1}, c_{2}\right)$ & $(0.01,0.02,0.56,-0.01,-0.01,-0.35)$ \\
$\left(b_{1}, a_{2}\right)\left(c_{1}, c_{2}\right)$ & $(0.01,0.02,0.56,-0.01,-0.02,-0.56)$ \\
$\left(b_{1}, b_{2}\right)\left(a_{1}, c_{2}\right)$ & $(0.12,0.02,0.56,-0.06,-0.01,-0.35)$ \\
$\left(b_{1}, b_{2}\right)\left(c_{1}, c_{2}\right)$ & $(0.02,0.02,0.56,-0.02,-0.03,-0.56)$ \\
$\left(c_{1}, a_{2}\right)\left(d_{1}, c_{2}\right)$ & $(0.01,0.06,0.64,-0.01,-0.04,-0.64)$ \\
$\left(c_{1}, b_{2}\right)\left(d_{1}, c_{2}\right)$ & $(0.02,0.06,0.64,-0.02,-0.06,-0.64)$ \\
\hline
\end{tabular}

The bipolar single-valued neutrosophic competition graph obtained using this method is given in Fig.10 where, the solid lines indicate the part of bipolar single-valued neutrosophic competition graph obtained from $G_{\mathcal{C}\left(\overrightarrow{G_{1}}\right) * \square \mathcal{C}\left(\overrightarrow{G_{2}}\right)^{*}}$, the dotted lines represent the part $G^{\square}$.

The Cartesian product $\overrightarrow{G_{1}} \square \overrightarrow{G_{2}}$ of bipolar single-valued neutrosophic digraph $\overrightarrow{G_{1}}$ and $\overrightarrow{G_{1}}$ is shown in Fig.11. The bipolar single-valued neutrosophic out-neighbourhoods of $\overrightarrow{G_{1}} \square \overrightarrow{G_{2}}$ are calculated in Table 8.

The bipolar single-valued neutrosophic competition graph of $\overrightarrow{G_{1}} \square \overrightarrow{G_{2}}$ is shown in Fig.12. 


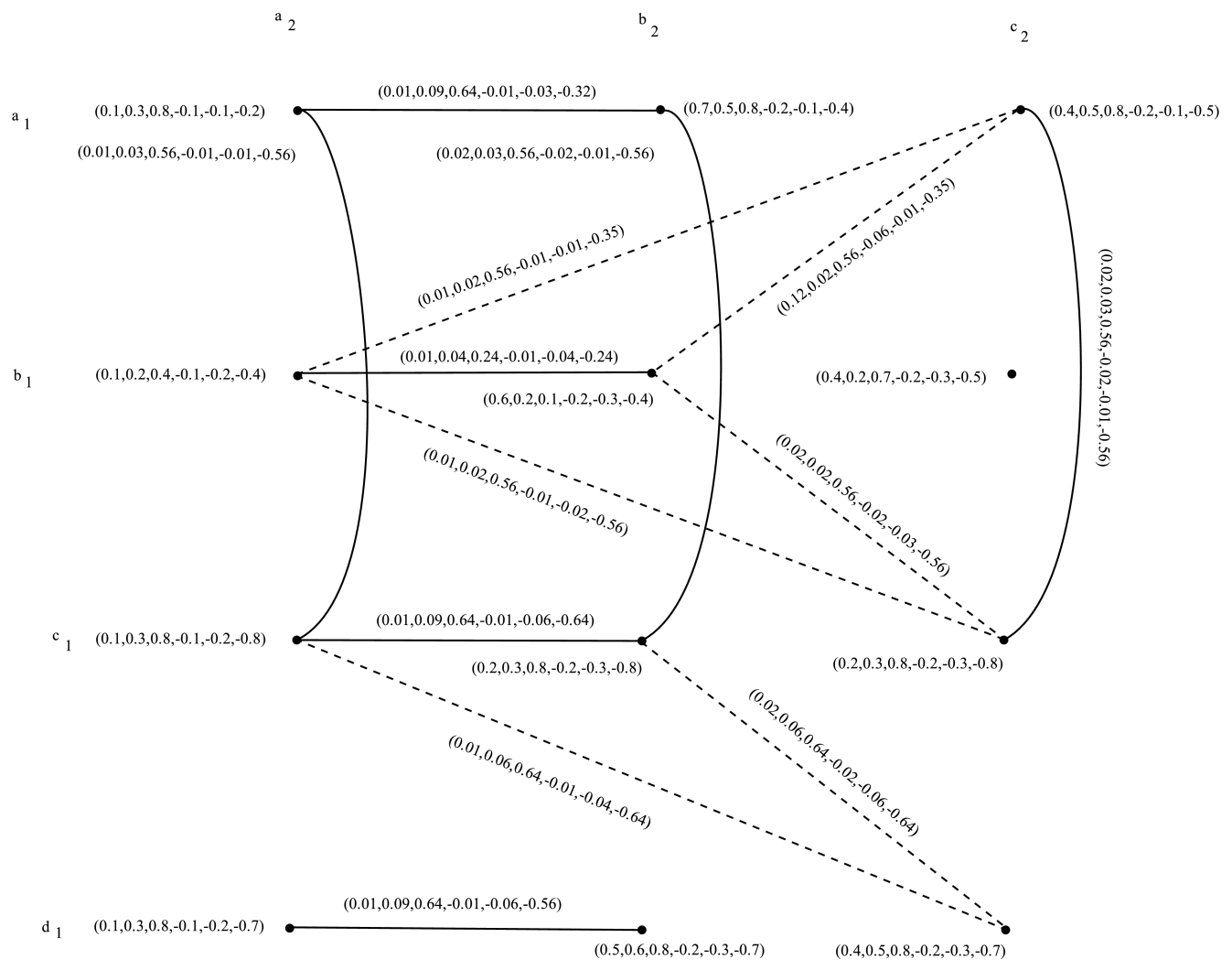

Figure 10: $G_{\mathcal{C}\left(\overrightarrow{G_{1}}\right) * \square \mathcal{C}\left(\overrightarrow{G_{2}}\right) *} \cup G^{\square}$

Table 8: Bipolar single-valued neutrosophic out-neighborhoods of $\overrightarrow{G_{1}} \square \overrightarrow{G_{2}}$

\begin{tabular}{|l|l|}
\hline$(x, y)$ & $\mathcal{N}^{+}(x, y)$ \\
\hline$\left(a_{1}, a_{2}\right)$ & $\left\{\left(\left(a_{1}, c_{2}\right), 0.1,0.3,0.8,-0.1,-0.1,-0.2\right),\left(\left(b_{1}, a_{2}\right), 0.1,0.1,0.7,-0.1,-0.1,-0.3\right)\right\}$ \\
$\left(a_{1}, b_{2}\right)$ & $\left\{\left(\left(a_{1}, c_{2}\right), 0.3,0.4,0.5,-0.1,-0.1,-0.4\right),\left(\left(b_{1}, b_{2}\right), 0.5,0.1,0.7,-0.1,-0.1,-0.4\right)\right\}$ \\
$\left(a_{1}, c_{2}\right)$ & $\left\{\left(\left(b_{1}, c_{2}\right), 0.4,0.1,0.7,-0.1,-0.1,-0.5\right)\right\}$ \\
$\left(b_{1}, a_{2}\right)$ & $\left\{\left(\left(b_{1}, c_{2}\right), 0.1,0.2,0.6,-0.1,-0.1,-0.4\right)\right\}$ \\
$\left(b_{1}, b_{2}\right)$ & $\left\{\left(\left(b_{1}, c_{2}\right), 0.3,0.2,0.5,-0.1,-0.2,-0.4\right)\right\}$ \\
$\left(b_{1}, c_{2}\right)$ & $\emptyset$ \\
$\left(c_{1}, a_{2}\right)$ & $\left\{\left(\left(c_{1}, c_{2}\right), 0.1,0.3,0.8,-0.1,-0.1,-0.8\right),\left(\left(b_{1}, a_{2}\right), 0.1,0.1,0.7,-0.1,-0.2,-0.7\right)\right\}$ \\
$\left(c_{1}, b_{2}\right)$ & $\left\{\left(\left(b_{1}, b_{2}\right), 0.1,0.1,0.7,-0.1,-0.2,-0.7\right),\left(\left(c_{1}, c_{2}\right), 0.2,0.3,0.8,-0.1,-0.2,-0.8\right)\right\}$ \\
$\left(c_{1}, c_{2}\right)$ & $\left\{\left(\left(b_{1}, c_{2}\right), 0.1,0.1,0.7,-0.1,-0.2,-0.7\right)\right\}$ \\
$\left(d_{1}, a_{2}\right)$ & $\left\{\left(\left(d_{1}, c_{2}\right), 0.1,0.3,0.8,-0.1,-0.1,-0.7\right),\left(\left(c_{1}, a_{2}\right), 0.1,0.2,0.7,-0.1,-0.2,-0.6\right)\right\}$ \\
$\left(d_{1}, b_{2}\right)$ & $\left.\left\{\left(\left(d_{1}, c_{2}\right), 0.3,0.4,0.8,-0.1,-0.2,-0.7\right),\left(c_{1}, b_{2}\right), 0.1,0.2,0.7,-0.1,-0.3,-0.6\right)\right\}$ \\
$\left(d_{1}, c_{2}\right)$ & $\left\{\left(\left(c_{1}, c_{2}\right), 0.1,0.2,0.7,-0.1,-0.3,-0.6\right)\right\}$ \\
\hline
\end{tabular}

It is clear from Figs. 10 and 12 that $G_{\mathcal{C}\left(\overrightarrow{G_{1}}\right)^{*} \square \mathcal{C}\left(\overrightarrow{G_{2}}\right)^{*}} \cup G^{\square} \cong \mathcal{C}\left(\overrightarrow{G_{1}} \square \overrightarrow{G_{2}}\right)$. 


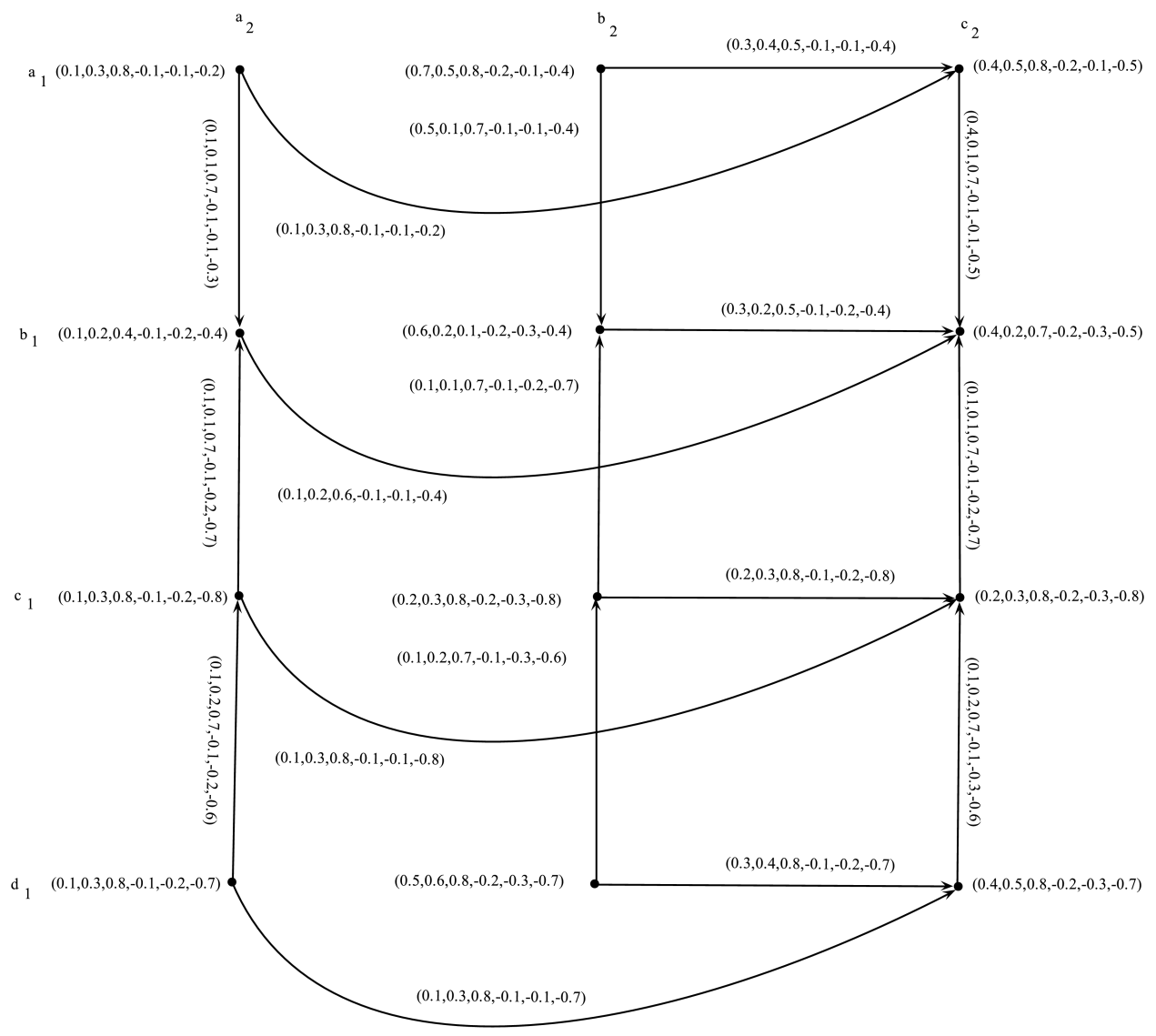

Figure 11: $\overrightarrow{G_{1}} \square \overrightarrow{G_{2}}$

\section{$\S 3$ Bipolar Single-Valued Neutrosophic Economic Competition Graphs}

In this section, we will use the following notations:

$\mathcal{P}_{x, y}^{m}$ : A bipolar single-valued neutrosophic path of length $m$ from $x$ to $y$.

$\overrightarrow{\mathcal{P}}_{x, y}^{m}$ : A directed bipolar single-valued neutrosophic path of length $m$ from $x$ to $y$.

$\mathcal{N}_{m}^{+}(x): m$-step bipolar single-valued neutrosophic out-neighborhood of vertex $x$.

$\mathcal{N}_{m}^{-}(x): m$-step bipolar single-valued neutrosophic in-neighborhood of vertex $x$.

$E \overrightarrow{(G)}$ : Bipolar single-valued neutrosophic economic competition graph of the bipolar singlevalued neutrosophic digraph $\vec{G}$.

$E_{m} \overrightarrow{(G)}: m$-step bipolar single-valued neutrosophic economic competition graph of the bipolar single-valued neutrosophic digraph $\vec{G}$. 


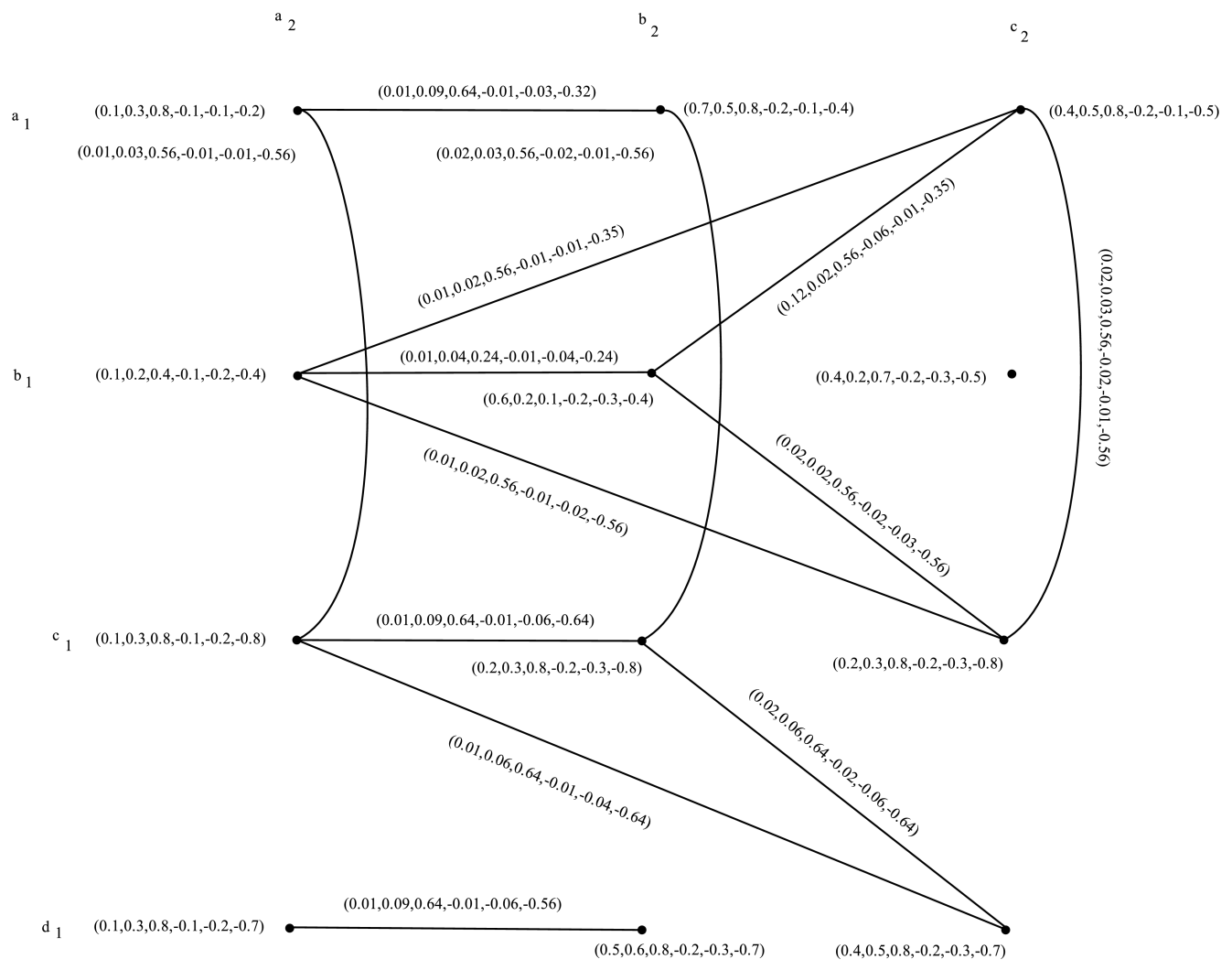

Figure 12: $\mathcal{C}\left(\overrightarrow{G_{1}} \square \overrightarrow{G_{2}}\right)$

Definition 3.1. The bipolar single-valued neutrosophic $m$-step out-neighborhood of vertex $x$ of a bipolar single-valued neutrosophic digraph $\vec{G}=(A, \vec{B})$ is bipolar single-valued neutrosophic set

$$
\mathcal{N}_{m}^{+}(x)=\left(X_{x}^{+}, t_{x}^{(p)^{+}}, i_{x}^{(p)^{+}}, f_{x}^{(p)^{+}}, t_{x}^{(n)^{+}}, i_{x}^{(n)^{+}}, f_{x}^{(n)^{+}}\right), \quad \text { where }
$$

$X_{x}^{+}=\{y \mid$ there exists a directed bipolar single-valued neutrosophic path of length $m$ from $x$ to $\left.y, \vec{P}_{x, y}^{m}\right\}, t_{x}^{(p)^{+}}: X_{x}^{+} \rightarrow[0,1], i_{x}^{(p)^{+}}: X_{z}^{+} \rightarrow[0,1], f_{x}^{(p)^{+}}: X_{z}^{+} \rightarrow[0,1] t_{x}^{(n)^{+}}: X_{x}^{+} \rightarrow[-1,0]$, $i_{x}^{(n)^{+}}: X_{z}^{+} \rightarrow[-1,0], f_{x}^{(n)^{+}}: X_{z}^{+} \rightarrow[-1,0]$ are defined by $t_{x}^{(p)^{+}}=\min \left\{t^{p} \overrightarrow{\left(x_{1}, x_{2}\right.}\right),\left(x_{1}, x_{2}\right)$ is an

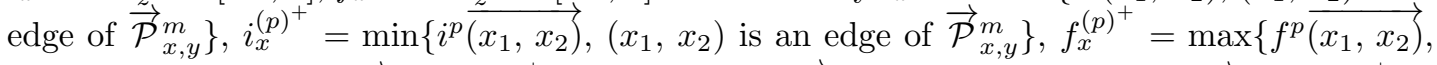
$\left(x_{1}, x_{2}\right)$ is an edge of $\left.\overrightarrow{\mathcal{P}}_{x, y}^{m}\right\}, t_{x}^{(n)^{+}}=\max \left\{t^{p} \overrightarrow{\left(x_{1}, x_{2}\right)},\left(x_{1}, x_{2}\right)\right.$ is an edge of $\left.\overrightarrow{\mathcal{P}}_{x, y}^{m}\right\}, i_{x}^{(n)^{+}}=$ $\max \left\{i^{n} \overrightarrow{\left(x_{1}, x_{2}\right)},\left(x_{1}, x_{2}\right)\right.$ is an edge of $\left.\overrightarrow{\mathcal{P}}_{x, y}^{m}\right\}, f_{x}^{(n)^{+}}=\min \left\{f^{n} \overrightarrow{\left(x_{1}, x_{2}\right)},\left(x_{1}, x_{2}\right)\right.$ is an edge of $\left.\overrightarrow{\mathcal{P}}_{x, y}^{m}\right\}$, respectively.

Example 3.1. Consider $\vec{G}=(A, \vec{B})$ is a bipolar single-valued neutrosophic digraph, such that $X=\{x, y, a, b, c, d\}$, as shown in Fig.13. Then, 2-step out-neighbourhood of vertices $x$, and $y$ is calculated as, $\mathcal{N}_{2}^{+}(x)=\{(b, 0.2,0.2,0.5,-0.2,-0.3,-0.3),(d, 0.2,0.2,0.5,-0.2,-0.3,-0.3)\}$, $\mathcal{N}_{2}^{+}(y)=\{(b, 0.1,0.3,0.2,-0.2,-0.3,-0.6),(d, 0.3,0.5,0.6,-0.2,-0.3,-0.5)\}$. 


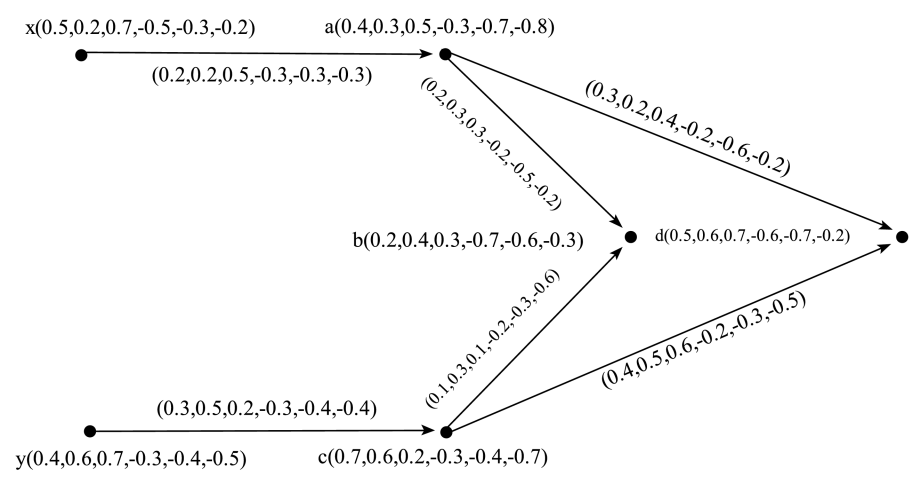

Figure 13: Bipolar single-valued neutrosophic digraph

Definition 3.2. The bipolar single-valued neutrosophic $m$-step in-neighborhood of vertex $x$ of a bipolar single-valued neutrosophic digraph $\vec{G}=(A, \vec{B})$ is bipolar single-valued neutrosophic set

$$
\mathcal{N}_{m}^{-}(x)=\left(X_{x}^{-}, t_{x}^{(p)^{-}}, i_{x}^{(p)^{-}}, f_{x}^{(p)^{-}}, t_{x}^{(n)^{-}}, i_{x}^{(n)^{-}}, f_{x}^{(n)^{-}}\right), \quad \text { where }
$$

$X_{x}^{-}=\{y \mid$ there exists a directed bipolar single-valued neutrosophic path of length $m$ from $y$ to $\left.x, \vec{P}_{y, x}^{m}\right\}, t_{x}^{(p)^{-}}: X_{x}^{-} \rightarrow[0,1], i_{x}^{(p)^{-}}: X_{z}^{-} \rightarrow[0,1], f_{x}^{(p)^{-}}: X_{z}^{-} \rightarrow[0,1] t_{x}^{(n)^{-}}: X_{x}^{-} \rightarrow[-1,0]$, $i_{x}^{(n)^{-}}: X_{z}^{-} \rightarrow[-1,0], f_{x}^{(n)^{-}}: X_{z}^{-} \rightarrow[-1,0]$ are defined by $t_{x}^{(p)^{-}}=\min \left\{t^{p} \overrightarrow{\left(x_{1}, x_{2}\right.}\right),\left(x_{1}, x_{2}\right)$ is an edge of $\left.\overrightarrow{\mathcal{P}}_{y, x}^{m}\right\}, i_{x}^{(p)^{-}}=\min \left\{i^{p} \overrightarrow{\left(x_{1}, x_{2}\right.}\right),\left(x_{1}, x_{2}\right)$ is an edge of $\left.\overrightarrow{\mathcal{P}}_{y, x}^{m}\right\}, f_{x}^{(p)^{-}}=\max \left\{f^{p}\left(\overrightarrow{x_{1}, x_{2}}\right)\right.$, $\left(x_{1}, x_{2}\right)$ is an edge of $\left.\overrightarrow{\mathcal{P}}_{y, x}^{m}\right\}, t_{x}^{(n)^{-}}=\max \left\{t^{n} \overrightarrow{\left(x_{1}, x_{2}\right.}\right),\left(x_{1}, x_{2}\right)$ is an edge of $\left.\overrightarrow{\mathcal{P}}_{y, x}^{m}\right\}, i_{x}^{(n)^{-}}=$ $\max \left\{i^{n} \overrightarrow{\left(x_{1}, x_{2}\right.}\right),\left(x_{1}, x_{2}\right)$ is an edge of $\left.\overrightarrow{\mathcal{P}}_{y, x}^{m}\right\}, f_{x}^{(n)^{-}}=\min \left\{f^{n} \overrightarrow{\left(x_{1}, x_{2}\right.}\right),\left(x_{1}, x_{2}\right)$ is an edge of $\left.\overrightarrow{\mathcal{P}}{ }_{y, x}^{m}\right\}$, respectively.

Example 3.2. Consider $\vec{G}=(A, \vec{B})$ is a bipolar single-valued neutrosophic digraph, such that, $X=\{a, b, c, d, e, f\}$, as shown in Fig.14. Then, 2-step in-neighborhood of vertices $a$, and $b$ is calculated as, $\mathcal{N}_{2}^{-}(a)=\{(f, 0.1,0.1,0.5,-0.1,-0.2,-0.6),(e, 0.3,0.1,0.7,-0.1,-0.2$, $-0.4)\}, \mathcal{N}_{2}^{-}(b)=\{(f, 0.1,0.3,0.6,-0.3,-0.4,-0.7),(e, 0.4,0.3,0.6,-0.3,-0.4,-0.5)\}$.

We now introduce the concepts of bipolar single-valued neutrosophic economic competition graphs and $m$-step bipolar single-valued neutrosophic economic competition graphs.

Due to the improvement of internet, people can transfer money from one place to another in a very short time. During the transfer of money, there are sources and destinations. Let us assume that there are four projects $P_{1}, P_{2}, P_{3}, P_{4}$ and again each of five institutions $I_{1}, I_{2}$, $I_{3}, I_{4}, I_{5}$, has to complete a project. Now, the institutions, projects and their relations make a digraph in which institutions and projects will be considered as vertices and the relations make directed edges. If two institutions are under the same project, then there will be an edge between them and so on. This kind of graph is called economic competition graph. Bipolar single-valued neutrosophic graph representation is more efficient to represent the competitions. 


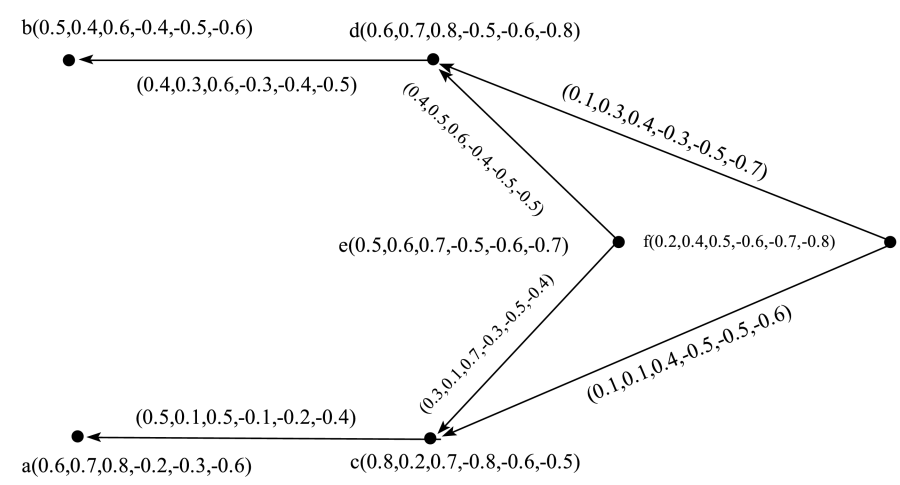

Figure 14: Bipolar single-valued neutrosophic digraph

Here, we introduce bipolar single-valued neutrosophic economic competition graphs and $m$-step bipolar single-valued neutrosophic economic competition graphs.

Definition 3.3. A bipolar single-valued neutrosophic economic competition graph of a bipolar single-valued neutrosophic digraph $\vec{G}=(A, \vec{B})$ is an undirected bipolar single-valued neutrosophic graph $E \overrightarrow{(G)}=(A, S)$ which has the same vertex set as in $\vec{G}$ and there is an edge between two vertices $x$ and $y$ if and only if $\mathcal{N}^{-}(x) \cap \mathcal{N}^{-}(y)$ is non-empty. The positive truthmembership, indeterminacy-membership, falsity-membership and negative truth-membership, indeterminacy-membership, falsity-membership values of the edge $(x, y)$ are defined as,

1. $t_{S}^{P}(x, y)=\left(t_{A}^{p}(x) \wedge t_{A}^{p}(y)\right) h_{1}\left(\mathcal{N}^{-}(x) \cap \mathcal{N}^{-}(y)\right)$,

2. $i_{S}^{p}(x, y)=\left(i_{A}^{p}(x) \wedge i_{A}^{p}(y)\right) h_{2}\left(\mathcal{N}^{-}(x) \cap \mathcal{N}^{-}(y)\right)$,

3. $f_{S}^{p}(x, y)=\left(f_{A}^{p}(x) \vee f_{A}^{p}(y)\right) h_{3}\left(\mathcal{N}^{-}(x) \cap \mathcal{N}^{-}(y)\right)$,

4. $t_{S}^{n}(x, y)=\left(t_{A}^{n}(x) \vee t_{A}^{n}(y)\right) h_{4}\left(\mathcal{N}^{-}(x) \cap \mathcal{N}^{-}(y)\right)$,

5. $i_{S}^{n}(x, y)=\left(i_{A}^{n}(x) \vee i_{A}^{n}(y)\right) h_{5}\left(\mathcal{N}^{-}(x) \cap \mathcal{N}^{-}(y)\right)$

6. $f_{S}^{n}(x, y)=\left(f_{A}^{n}(x) \wedge f_{A}^{n}(y)\right) h_{6}\left(\mathcal{N}^{-}(x) \cap \mathcal{N}^{-}(y)\right)$,

for all $x, y \in X$.

Example 3.3. Consider $\vec{G}=(A, \vec{B})$ is a bipolar single-valued neutrosophic digraph, such that, $X=\{a, b, c, d, e, f, g\}$, as shown in Fig.15. Then, $\mathcal{N}^{-}(a)=\emptyset, \mathcal{N}^{-}(b)=\{(a, 0.5$, $0.6,0.4,-0.1,-0.2,-0.5)\}, \mathcal{N}^{-}(c)=\{(a, 0.5,0.7,0.5,-0.5,-0.2,-0.1)\}, \mathcal{N}^{-}(d)=\{(a$, $0.5,0.4,0.4,-0.1,-0.1,-0.1)\}, \mathcal{N}^{-}(e)=\{(c, 0.6,0.6,0.5,-0.4,-0.5,-0.5),(b, 0.6,0.7$, $0.6,-0.1,-0.4,-0.6)\}, \mathcal{N}^{-}(f)=\{(d, 0.8,0.5,0.7,-0.1,-0.1,-0.1),(c, 0.6,0.6,0.6,-0.3$, $-0.1,-0.1)\}$, and $\mathcal{N}^{-}(g)=\{(d, 0.6,0.4,0.6,-0.1,-0.2,-0.5)\}$. After calculating the nonempty intersections of in-neighborhoods of the vertices, we have Fig.16 representing bipolar single-valued neutrosophic economic competition graph.

Definition 3.4. Suppose $\vec{G}=(A, \vec{B})$ is a bipolar single-valued neutrosophic digraph. The $m$-step bipolar single-valued neutrosophic economic competition graph of bipolar single-valued 


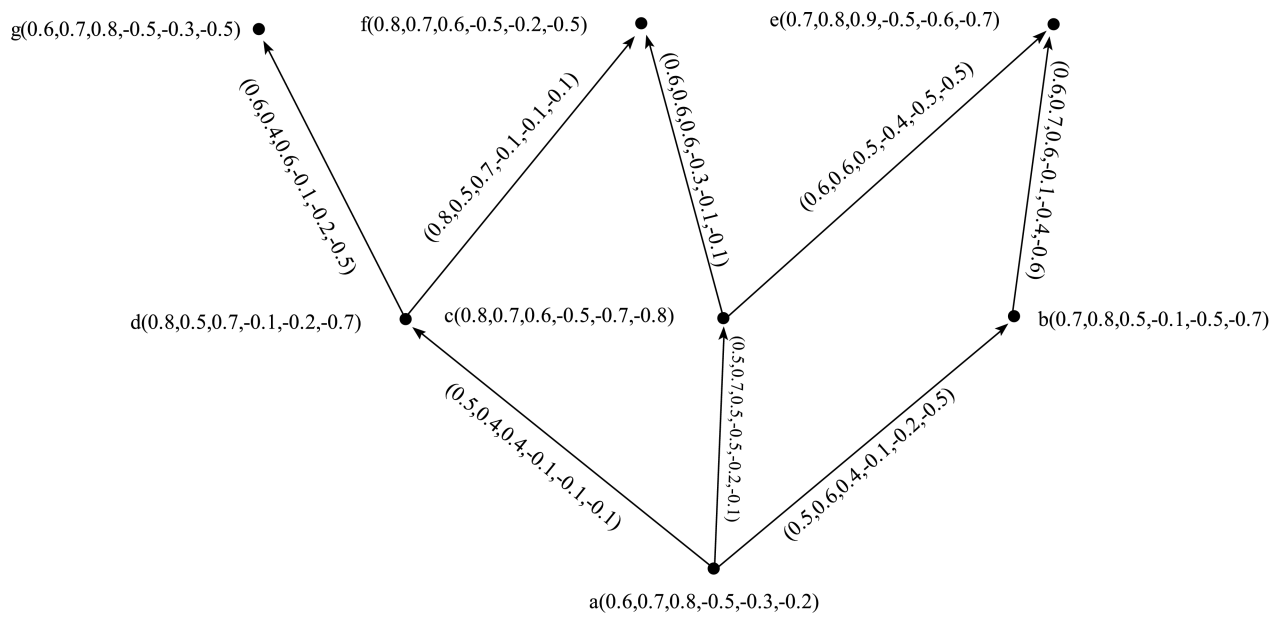

Figure 15: Bipolar single-valued neutrosophic digraph
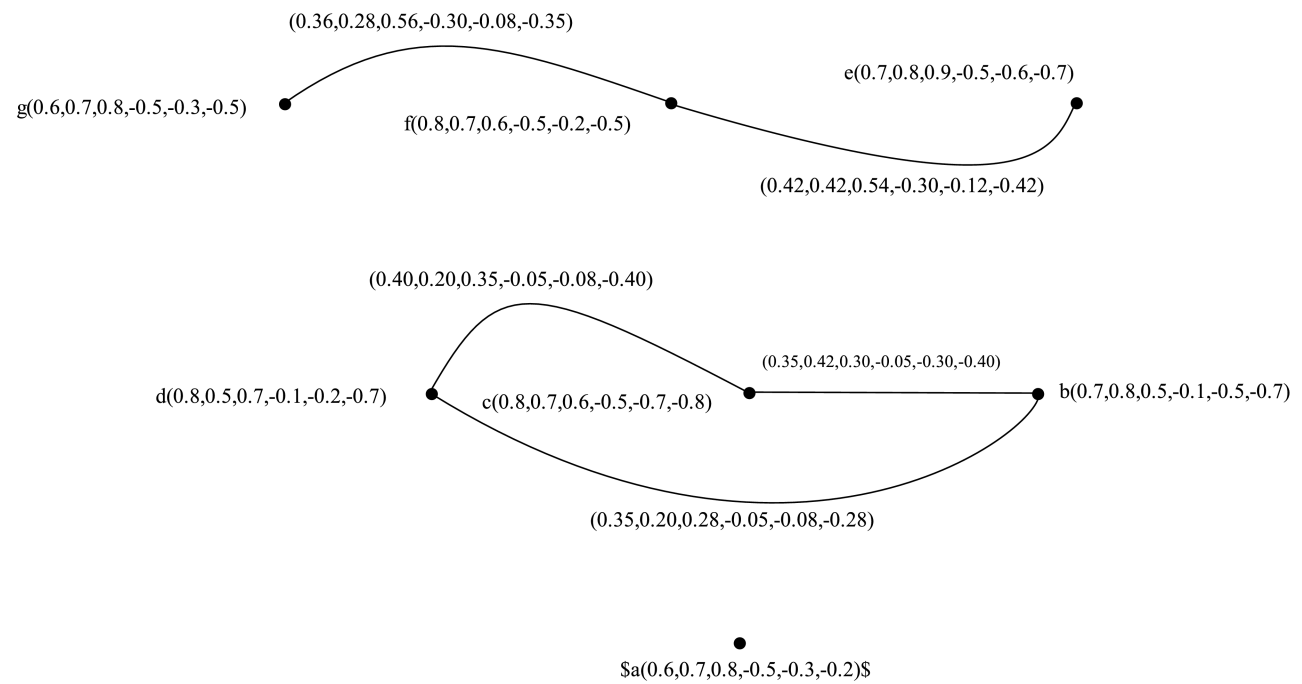

Figure 16: Bipolar single-valued neutrosophic economic competition graph

neutrosophic digraph $\vec{G}$ is denoted by $E_{m}(\vec{G})=(A, S)$ which has same bipolar single-valued neutrosophic set of vertices as in $\vec{G}$ and has an edge between two vertices $x, y \in X$ in $E_{m}(\vec{G})$ if and only if $\left(\mathcal{N}_{m}^{-}(x) \cap \mathcal{N}_{m}^{-}(y)\right)$ is a non-empty bipolar single-valued neutrosophic set in $\vec{G}$. The positive truth-membership value of edge $(x, y)$ in $E_{m}(\vec{G})$ is $t_{S}^{p}(x, y)=$ $\left[t_{A}^{p}(x) \wedge t_{A}^{p}(z)\right] h_{1}\left(\mathcal{N}_{m}^{-}(x) \cap \mathcal{N}_{m}^{-}(y)\right)$, the positive indeterminacy-membership value of edge $(x, y)$ in $E_{m}(\vec{G})$ is $i_{S}^{p}(x, y)=\left[i_{A}^{p}(x) \wedge i_{A}^{p}(y)\right] h_{2}\left(\mathcal{N}_{m}^{-}(x) \cap \mathcal{N}_{m}^{-}(y)\right)$, the positive falsity-membership value of edge $(x, y)$ in $E_{m}(\vec{G})$ is $f_{S}^{p}(x, y)=\left[f_{A}^{p}(x) \vee f_{A}^{p}(y)\right] h_{3}\left(\mathcal{N}_{m}^{-}(x) \cap \mathcal{N}_{m}^{-}(y)\right)$, the negative truthmembership value of edge $(x, y)$ in $E_{m}(\vec{G})$ is $t_{S}^{n}(x, y)=\left[t_{A}^{n}(x) \vee t_{A}^{n}(z)\right] h_{4}\left(\mathcal{N}_{m}^{-}(x) \cap \mathcal{N}_{m}^{-}(y)\right)$, the negative indeterminacy-membership value of edge $(x, y)$ in $E_{m}(\vec{G})$ is $i_{S}^{n}(x, y)=\left[i_{A}^{n}(x) \vee\right.$ 
$\left.i_{A}^{n}(y)\right] h_{5}\left(\mathcal{N}_{m}^{-}(x) \cap \mathcal{N}_{m}^{-}(y)\right)$, the negative falsity-membership value of edge $(x, y)$ in $E_{m}(\vec{G})$ is $f_{S}^{n}(x, y)=\left[f_{A}^{n}(x) \wedge f_{A}^{n}(y)\right] h_{6}\left(\mathcal{N}_{m}^{-}(x) \cap \mathcal{N}_{m}^{-}(y)\right)$.

The 2-step bipolar single-valued neutrosophic economic competition graph is illustrated by the following example.

Example 3.4. Consider $\vec{G}=(A, \vec{B})$ is a bipolar single-valued neutrosophic digraph, such that, $X=\{a, b, c, d, e, f\}$, as shown in Fig.17. Then, we calculate $\mathcal{N}_{2}^{-}(a)=\{(f, 0.1,0.1$, $0.5,-0.1,-0.2,-0.6),(e, 0.3,0.1,0.7,-0.1,-0.2,-0.4)\}, \mathcal{N}_{2}^{-}(b)=\{(f, 0.1,0.3,0.6,-0.3$, $-0.4,-0.7),(e, 0.4,0.3,0.6,-0.3,-0.4,-0.5)\}$, and $\mathcal{N}_{2}^{-}(a) \cap \mathcal{N}_{2}^{-}(b)=\{(f, 0.1,0.1,0.6,-0.1$, $-0.2,-0.7),(e, 0.3,0.1,0.7,-0.1,-0.2,-0.5)\}$. 2-step bipolar single-valued neutrosophic economic competition graph is obtained, as shown in Fig.18.

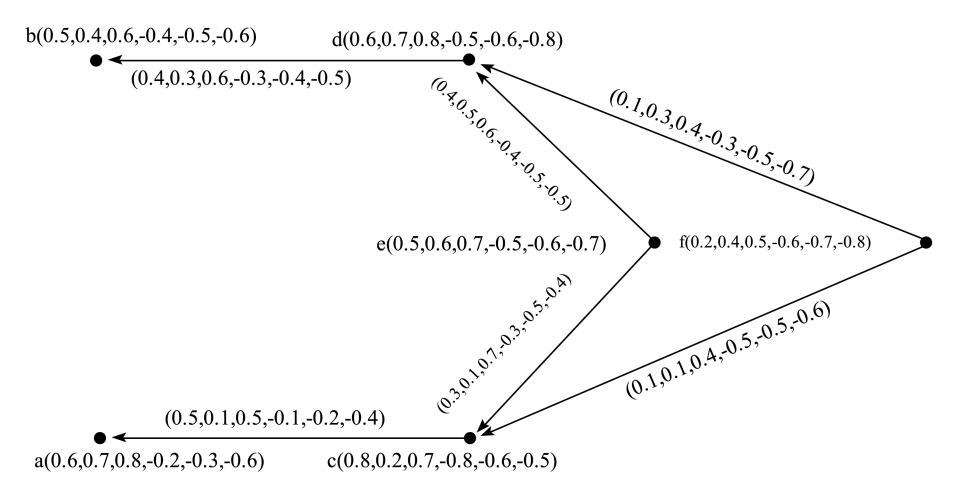

Figure 17: Bipolar single-valued neutrosophic digraph

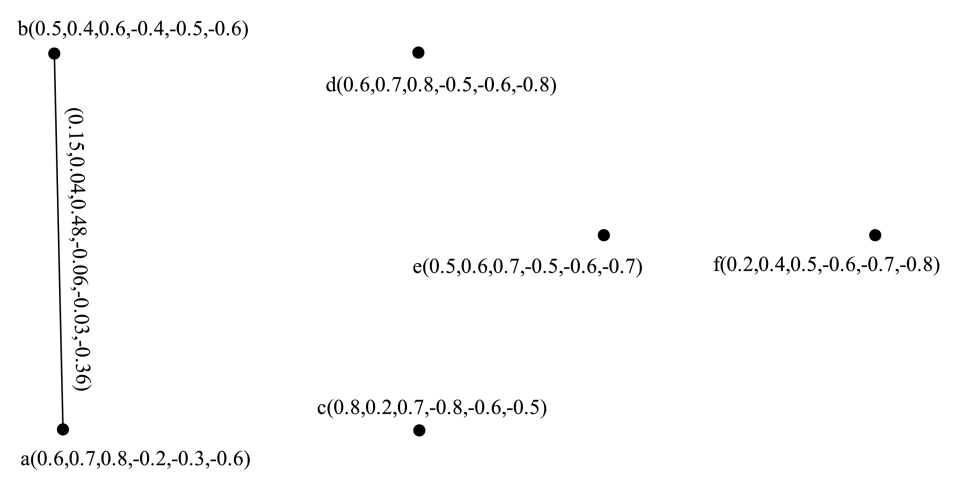

Figure 18: 2-step bipolar single-valued neutrosophic economic competition graph

We state the following theorems without proofs.

Theorem 3.1. Bipolar single-valued neutrosophic competition graph and bipolar single-valued neutrosophic economic competition graph of any complete bipolar single-valued neutrosophic digraph are same. 
Theorem 3.2. If $\overrightarrow{G_{1}}$ is the bipolar single-valued neutrosophic sub-digraph of $\vec{G}$. Then

1. $\mathcal{C}_{m}\left(\overrightarrow{G_{1}}\right) \subset \mathcal{C}_{m}(\vec{G})$.

2. $E_{m}\left(\overrightarrow{G_{1}}\right) \subset E_{m}(\vec{G})$.

\section{$\S 4$ Applications}

Competition graphs are very important to represent the competition between objects. But still these representations are unsuccessful to deal with all the competitions of world, for that purpose bipolar single-valued neutrosophic competition graphs are introduced. Now, we discuss applications of bipolar single-valued neutrosophic competition graphs to study the competition along with algorithms. The bipolar single-valued neutrosophic competition graphs have many utilizations in different areas.

1. Organizational Designations Competition. Consider the bipolar single-valued neutrosophic digraph as shown in Fig.19 representing the competition between applicants for designations in an organization. Let \{Angus, Alvin, Alina, Colin, Alma\} be the set of applicants for the designations \{Board of director(BOD), CEO, Director of marketing(DOM), Director of human resources(DOHR)\}. The positive degree of membership $t^{p}(x)$ of each applicant represent the percentage of hardwork towards the goals of organization, $i^{p}(x)$ and $f^{p}(x)$ represent the indeterminacy and falsity in this percentage. The negative degree of membership $t^{n}(x)$ represents the percentage that the applicant is not effective in order to fulfill the goals of that organization, $i^{n}(x)$ and $f^{n}(x)$ represent the indeterminacy and falsity in this percentage. The positive degree of membership $t^{p}(x)$ of each directed edge between applicants and designations represent the percentage of eligibility for that designation in organization, $i^{p}(x)$ and $f^{p}(x)$ represent the indeterminacy and falsity in this percentage. The negative degree of membership $t^{n}(x)$ of each directed edge between applicants and designations represent the percentage of non-eligibility for that designation in organization, $i^{n}(x)$ and $f^{n}(x)$ represent the indeterminacy and falsity in this percentage. The bipolar single-valued neutrosophic competition graph can be used in order to find the designations of the applicants.

Table 9: Bipolar single-valued neutrosophic out-neighbourhoods

\begin{tabular}{|l|l|}
\hline$x \in X$ & $\mathcal{N}^{+}(x)$ \\
\hline Angus & $\{(B O D, 0.6,0.4,0.5,-0.1,-0.5,-0.5),(C E O, 0.6,0.4,0.5,-0.1,-0.5,-0.7)$, \\
& $(D O M, 0.6,0.4,0.2,-0.1,-0.5,-0.4)\}$ \\
Alma & $\{(D O H R, 0.3,0.4,0.6,-0.4,-0.3,-0.5)\}$ \\
Alvin & $\{(D O H R, 0.2,0.4,0.6,-0.5,-0.4,-0.6),(C E O, 0.2,0.4,0.5,-0.5,-0.6,-0.5)\}$ \\
Colin & $\{(D O H R, 0.4,0.6,0.5,-0.2,-0.3,-0.4),(D O M, 0.4,0.5,0.5,-0.2,-0.3,-0.4)\}$ \\
Alina & $\{(B O D, 0.5,0.5,0.6,-0.1,-0.4,-0.4),(C E O, 0.5,0.5,0.5,-0.4,-0.5,-0.4)$, \\
& $(D O M, 0.4,0.4,0.4,-0.3,-0.4,-0.2)\}$ \\
&
\end{tabular}

Therefore, $\mathcal{N}^{+}($Angus $) \cap \mathcal{N}^{+}($Alma $)=\emptyset, \mathcal{N}^{+}($Alma $) \cap \mathcal{N}^{+}($Alina $)=\emptyset, \mathcal{N}^{+}$(Angus $) \cap$ 


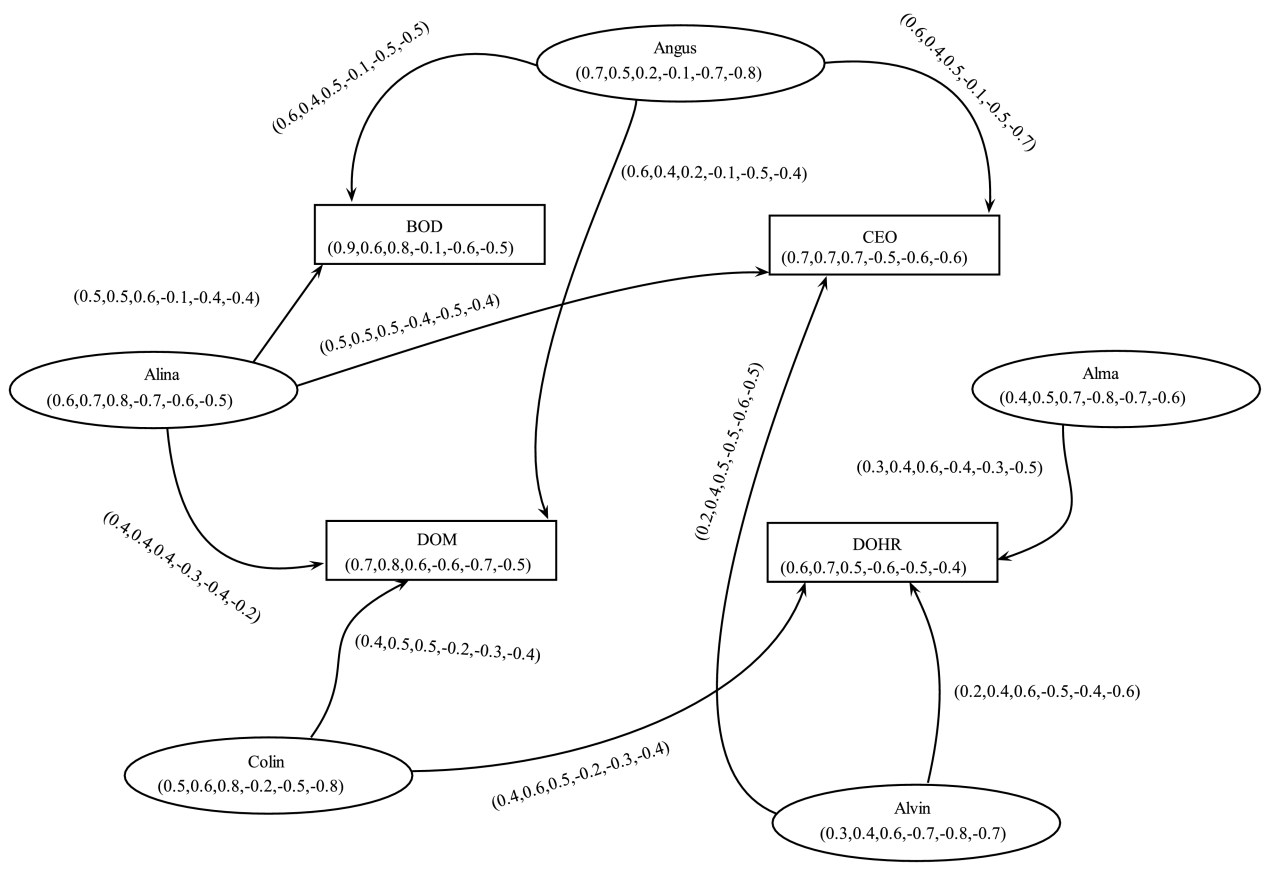

Figure 19: Bipolar single-valued neutrosophic digraph

$\mathcal{N}^{+}($Alvin $)=\{($CEO $0.2,0.4,0.5,-0.1,-0.5,-0.7)\}, \mathcal{N}^{+}($Angus $) \cap \mathcal{N}^{+}($Colin $)=\{($DOM, $0.4,0.4,0.5,-0.1,-0.3,-0.4)\}, \mathcal{N}^{+}($Alma $) \cap \mathcal{N}^{+}($Alvin $)=\{(D O H R, 0.2,0.4,0.6,-0.4,-0.3$, $-0.6)\}, \mathcal{N}^{+}($Alma $) \cap \mathcal{N}^{+}($Colin $)=\{($DOHR $, 0.3,0.4,0.6,-0.2,-0.3,-0.5)\}, \mathcal{N}^{+}($Alvin $) \cap$ $\mathcal{N}^{+}($Colin $)=\{($DOHR $, 0.2,0.4,0.6,-0.2,-0.3,-0.6)\}, \mathcal{N}^{+}($Alvin $) \cap \mathcal{N}^{+}($Alina $)=\{(C E O$, $0.2,0.4,0.5,-0.4,-0.5,-0.5)\}, \mathcal{N}^{+}($Colin $) \cap \mathcal{N}^{+}($Alina $)=\{(D O M, 0.4,0.4,0.5,-0.2,-0.3$, $-0.4)\}, \mathcal{N}^{+}($Angus $) \cap \mathcal{N}^{+}($Alina $)=\{(B O D, 0.5,0.4,0.6,-0.1,-0.4,-0.5),(C E O, 0.5,0.4$, $0.5,-0.1,-0.5,-0.7),(D O M, 0.4,0.4,0.4,-0.1,-0.4,-0.2)\}$. The bipolar single-valued neutrosophic competition graph is shown in Fig.20. The solids lines indicate the strength of competition between two applicants and dashed lines indicate the applicant competing for the particular designation. For example, Angus and Alina both are competing for the designation, BOD and strength of competition between them is $(0.30,0.20$,

$0.32,-0.05,-0.24,-0.32)$. In Table 10, $T(y, d)$ represents the value of strength of competition of applicant $y$ for designation $d$ with respect to hardwork in order to fulfil the goals of that organization. The strength to compete the others applicant with respect to particular designation is calculated in Table 10 .

From Table 10, Angus and Alina has equal strength to compete the other for designation, BOD. Angus competes the others for the designations DOM and CEO, while, Colin competes the others for the designations DOHR. 


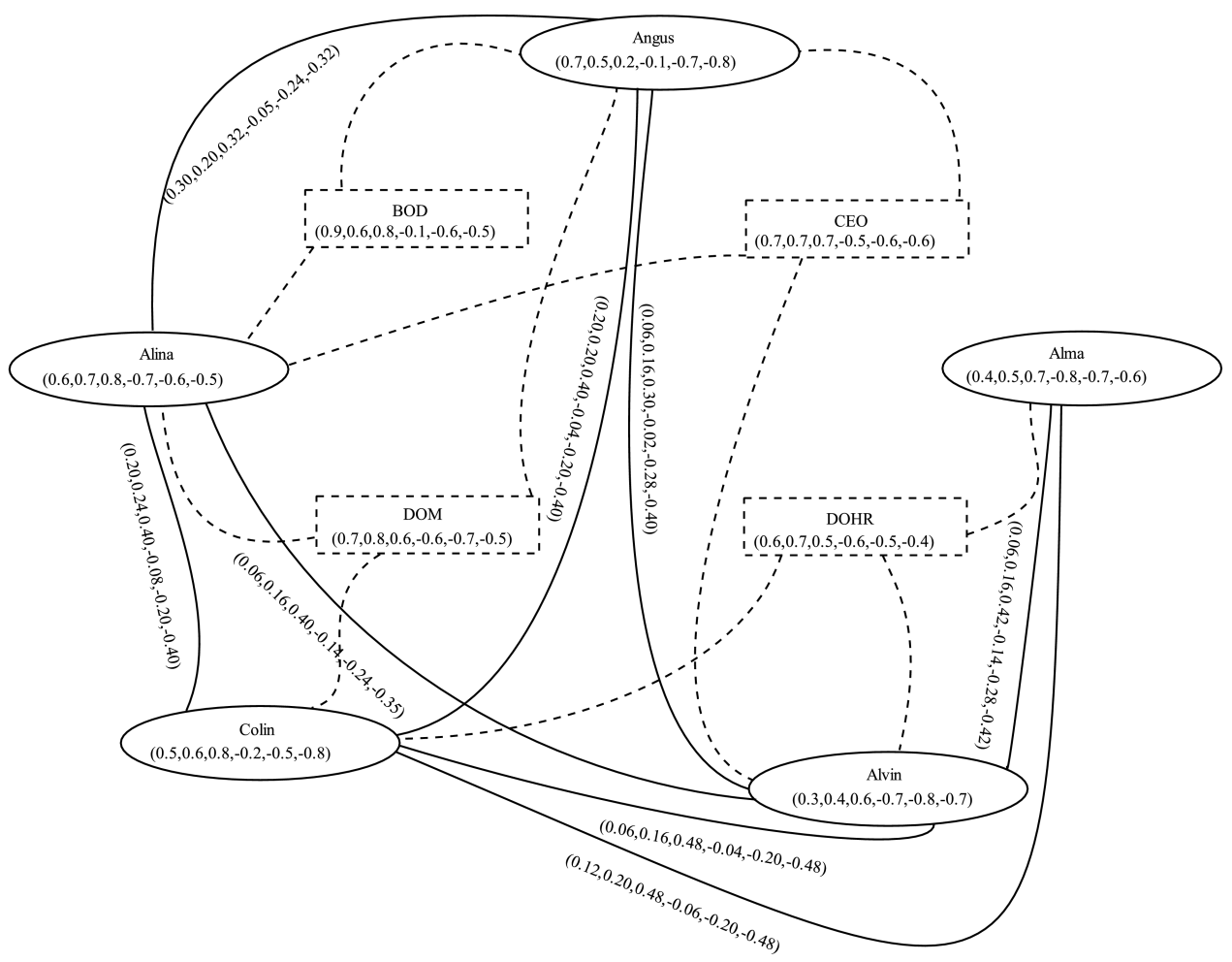

Figure 20: Corresponding bipolar single-valued neutrosophic competition graph

Table 10: Strength of competition of applicants for particular designations

\begin{tabular}{|l|l|l|l|}
\hline (Applicant, Designation) & In competition & $T$ (Applicant, Designation) & $S$ (Applicant, Designation) \\
\hline (Angus, BOD ) & Alina & $(0.30,0.20,0.32,-0.05,-0.24,-0.32)$ & 1.29 \\
(Alina, BOD) & Angus & $(0.30,0.20,0.32,-0.05,-0.24,-0.32)$ & 1.29 \\
\hline (Angus, DOM) & Alina, Colin & $(0.25,0.20,0.36,-0.045,-0.22,-0.36)$ & 1.225 \\
(Alina, DOM) & Angus, Colin & $(0.25,0.22,0.36,-0.065,-0.22,-0.36)$ & 1.185 \\
(Colin, DOM) & Angus, Alina & $(0.20,0.22,0.40,-0.060,-0.20,-0.40)$ & 1.120 \\
\hline (Angus, CEO) & Alina, Alvin & $(0.180,0.180,0.310,-0.035,-0.260,-0.360)$ & 1.275 \\
(Alina, CEO) & Angus, Alvin & $(0.180,0.180,0.360,-0.095,-0.24,-0.335)$ & 1.120 \\
(Alvin, CEO) & Angus, Alina & $(0.06,0.16,0.35,-0.080,-0.260,-0.375)$ & 1.105 \\
\hline (Alma, DOHR) & Alvin, Colin & $(0.09,0.180,0.450,-0.100,-0.240,-0.450)$ & 1.050 \\
(Alvin, DOHR) & Colin, Alma & $(0.06,0.16,0.450,-0.09,-0.240,-0.450)$ & 0.050 \\
(Colin, DOHR) & Alma, Alvin & $(0.09,0.180,0.480,-0.05,-0.20,-0.48)$ & 1.060 \\
\hline
\end{tabular}

We now elaborate this method by an algorithm.

\section{Algorithm}

Step 1. Input the positive truth, indeterminacy and falsity-memberships values for set of $q$ applicants.

Step 2. If for any two distinct vertices $y_{i}$ and $y_{j}, t^{p}\left(y_{i} y_{j}\right)>0, i^{p}\left(y_{i} y_{j}\right)>0, f^{p}\left(y_{i} y_{j}\right)>0$, 


$$
\begin{aligned}
t^{n}\left(y_{i} y_{j}\right)< & 0, i^{n}\left(y_{i} y_{j}\right)<0, f^{n}\left(y_{i} y_{j}\right)<0 \text {, then } \\
& \left(y_{j}, t^{p}\left(y_{i} y_{j}\right), i^{p}\left(y_{i} y_{j}\right), f^{p}\left(y_{i} y_{j}\right), t^{n}\left(y_{i} y_{j}\right), i^{n}\left(y_{i} y_{j}\right), f^{n}\left(y_{i} y_{j}\right)\right) \in \mathcal{N}^{+}\left(y_{i}\right) .
\end{aligned}
$$

Step 3. Repeat Step 2 for all vertices $y_{i}$ and $y_{j}$ to calculate bipolar single-valued neutrosophicout-neighbourhoods $\mathcal{N}^{+}\left(y_{i}\right)$.

Step 4. Calculate $\mathcal{N}^{+}\left(y_{i}\right) \cap \mathcal{N}^{+}\left(y_{j}\right)$ for each pair of distinct vertices $y_{i}$ and $y_{j}$.

Step 5. Calculate $h\left[\mathcal{N}^{+}\left(y_{i}\right) \cap \mathcal{N}^{+}\left(y_{j}\right)\right]$.

Step 6. If $\mathcal{N}^{+}\left(y_{i}\right) \cap \mathcal{N}^{+}\left(y_{j}\right) \neq \emptyset$ then draw an edge $y_{i} y_{j}$.

Step 7. Repeat step 6 for all pair of distinct vertices.

Step 8. Assign membership values to each edge $y_{i} y_{j}$ using the conditions

$$
\begin{array}{ll}
t^{p}\left(y_{i} y_{j}\right)=\left(y_{i} \wedge y_{j}\right) h_{1}\left[\mathcal{N}^{+}\left(y_{i}\right) \cap \mathcal{N}^{+}\left(y_{j}\right)\right] & t^{n}\left(y_{i} y_{j}\right)=\left(y_{i} \vee y_{j}\right) h_{4}\left[\mathcal{N}^{+}\left(y_{i}\right) \cap \mathcal{N}^{+}\left(y_{j}\right)\right] \\
i^{p}\left(y_{i} y_{j}\right)=\left(y_{i} \wedge y_{j}\right) h_{2}\left[\mathcal{N}^{+}\left(y_{i}\right) \cap \mathcal{N}^{+}\left(y_{j}\right)\right] & i^{n}\left(y_{i} y_{j}\right)=\left(y_{i} \vee y_{j}\right) h_{5}\left[\mathcal{N}^{+}\left(y_{i}\right) \cap \mathcal{N}^{+}\left(y_{j}\right)\right] \\
f^{p}\left(y_{i} y_{j}\right)=\left(y_{i} \vee y_{j}\right) h_{3}\left[\mathcal{N}^{+}\left(y_{i}\right) \cap \mathcal{N}^{+}\left(y_{j}\right)\right] & f^{n}\left(y_{i} y_{j}\right)=\left(y_{i} \wedge y_{j}\right) h_{6}\left[\mathcal{N}^{+}\left(y_{i}\right) \cap \mathcal{N}^{+}\left(y_{j}\right)\right]
\end{array}
$$

Step 9. If $y, r_{1}, r_{2}, r_{3}, \ldots, r_{q}$ are the applicants competing for designation $d$, then strength of competition $T(y, d)=\left(t^{p}(y, d), i^{p}(y, d), f^{p}(y, d), t^{n}(y, d), i^{n}(y, d), f^{n}(y, d)\right)$ of each applicant $y$ for the designation $d$ is

$$
\begin{aligned}
& T(y, d)=\frac{\left(t^{p}\left(y r_{1}\right)+\ldots+t^{p}\left(y r_{q}\right), i^{p}\left(y r_{1}\right)+\ldots+i^{p}\left(y r_{q}\right), f^{p}\left(y r_{1}\right)+\ldots+f^{p}\left(y r_{q}\right), t^{n}\left(y r_{1}\right)+\ldots+t^{n}\left(y r_{q}\right), i^{n}\left(y r_{1}\right)\right.}{q}+ \\
& \ldots+\frac{\left.i^{n}\left(y r_{q}\right), f^{n}\left(y r_{1}\right)+\ldots+f^{n}\left(y r_{q}\right)\right)}{q} .
\end{aligned}
$$

Step 10. Calculate $S(y, d)$, the strength of competition of each applicant $y$ for designation $d$.

$$
S(y, d)=t^{p}(y, d)-\left(i^{p}(y, d)+f^{p}(y, d)\right)+1+t^{n}(y, d)-\left(i^{n}(y, d)+f^{n}(y, d)\right) .
$$

2. Competition Between Different Brands. Brands are of great importance and value for both the buyer and the seller. Brands are centred on what the seller promises to deliver to its customers and the consumer's expectations of a particular product. A brand can be defined as a name, slogan or anything that can be used to identify and differentiate a particular product or service. Strong brands such as Stylo shoes and Borjan shoes evoke sound emotional and physiological responses from customers.

Branding has several benefits for sellers. By having brands for their products, sellers get product recognition and product differentiation. This means that consumers would be able to accentuate the value they receive from one seller's product in comparison with other products of a similar nature and they would also be able to easily spot these products among other products. Branding aligns the seller's advertising and promotional activities. It allows the seller to form emotional relationship with their customers which is important because people base their purchasing decisions mainly on emotions and not logic.

Within the business, brands serve as guidance for employees since they clearly dictate what the company product is about. Branding is also of value to sellers in terms of profitability. A 
product with a well-known brand attached to it would bring more revenue to a seller than a product without a brand. For example a cup of coffee without a brand can cost 70, however a cup of coffee with the Kaymu brand attached to it can cost 999. For the seller, a good brand increases the perceived value of its product and allows it to have a better price and better sales. For the buyer, a brand represents the satisfaction and value that he or she anticipates and desires from the process of buying and using a product or service. Brands often appeal to buyers emotionally this in turn helps build relationships between buyers and sellers. Brands provide buyers with value which is why they are often times prepared to pay more for branded products. Among buyers, brands are often a key factor behind purchasing decisions, people often gravitate toward brands that they are familiar with and that are trusted. In conclusion, brands exist as feelings and experiences that extend beyond the product or service which create relationships between the buyer and seller and have great value for both parties. We take the following brands in order to discuss the competition between them.

1. Deepak Perwani:

At the top of the list we would bring up the name of famous Deepak Perwani. He has been linked with the fashion designing career since the year 1994. He has been working out in offering with the women wear along with the men wear clothing collections as well. He has been best with his designing skills as he does add the dresses with the eastern and western images. This designer has even got his name listed in a record book as he made a kurta of 53 meter for a person.

2. Maria B:

On the 2nd spot of expensive clothing brands in Pakistan we would mention with Maria B. She is one of the top most wanted fashion designers of Pakistan. She has been infused in offering with the collection line of the bridal, casual and western wear. She has done her graduation from the Pakistan School Of Fashion Design and later she organized her company in the year 1999.

3. Khaadi:

Khaadi is one of the top most famous clothing brands of the Pakistan. Khaadi was started by Shamoon Sultan in 1998. This brand has been in the front line for the purpose of offering with the high quality of the dresses designs in the magnificent designing concepts. It does offer with the clothing collections for both men and women along with the kidswear.

4. Aamir Adnan:

On the 4th spot on our list we would bring up the name of designer brand Aamir Adnan. This brand is one of the top most wanted brands of clothing for the men. It has made its renowned name all through its valuable fashion of sherwani kurtas.

Consider the bipolar single-valued neutrosophic digraph as shown in Fig.21 representing the competition between brands. Let \{Deepak Perwani, Khaadi, Maria B, Aamir Adnan\} be the set of brands and \{Uniqueness, Passion, Consistency\}, these are the qualities for the popularity of a particular brand. The positive degree of membership $t^{p}(x)$ of each brand represents the percentage of productivity of traditional clothes towards the satisfaction of customer, $i^{p}(x)$ 
and $f^{p}(x)$ represent the indeterminacy and falsity in this percentage. The negative degree of membership $t^{n}(x)$ represents the percentage that the brand can not satisfy the customer, $i^{n}(x)$ and $f^{n}(x)$ represent the indeterminacy and falsity in this percentage. The positive degree of membership $t^{p}(x)$ of each directed edge between brands and the qualities represent the percentage of having that quality, $i^{p}(x)$ and $f^{p}(x)$ represent the indeterminacy and falsity in this percentage. The negative degree of membership $t^{n}(x)$ of each directed edge between brands and qualities represent the percentage of having no qualities, $i^{n}(x)$ and $f^{n}(x)$ represent the indeterminacy and falsity in this percentage. Thus, bipolar single-valued neutrosophic competition graph can be used in order to find competitions between different brands.

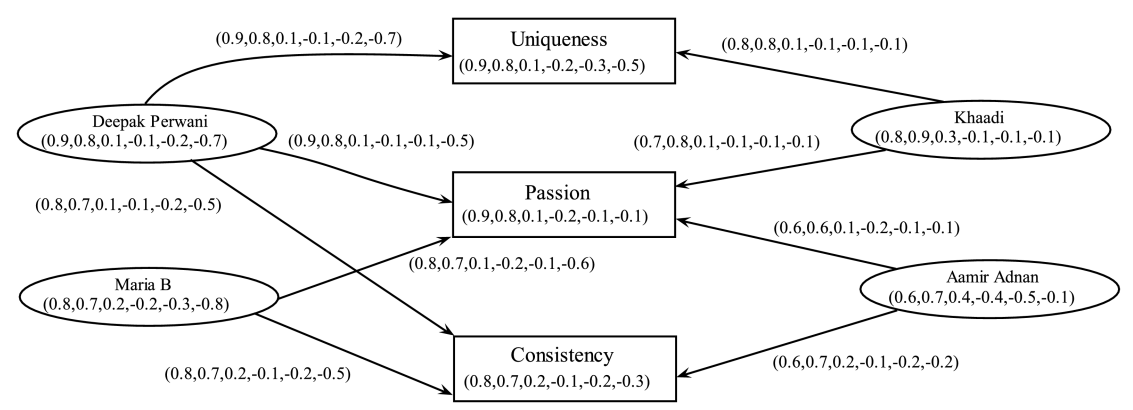

Figure 21: Bipolar single-valued neutrosophic digraph

Table 11: Bipolar single-valued neutrosophic out-neighbourhoods

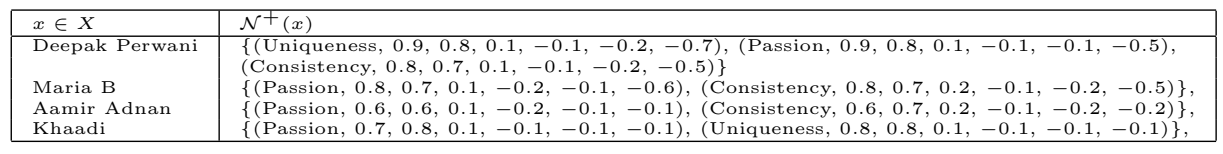

The bipolar single-valued neutrosophic competition graph is shown in Fig.22. The solid lines indicate the strength of competition between two brands and dashed lines indicate the brands are competing for the particular quality. For example, Deepak Perwani and Khaadi both are competing for the quality, uniqueness and strength of competition between them is $(0.64,0.64,0.03,-0.08,-0.08,-0.07)$. In Table $12, T(y, d)$ represents the value of strength of competition of brand $y$ for quality $d$ with respect to level of satisfaction for the customer.

From Table 12, Deepak Perwani and Khaadi has equal strength to compete with the others for quality, uniqueness. Deepak Perwani competes with the others for the quality passion, while, Maria B competes with the others for the quality consistency. 


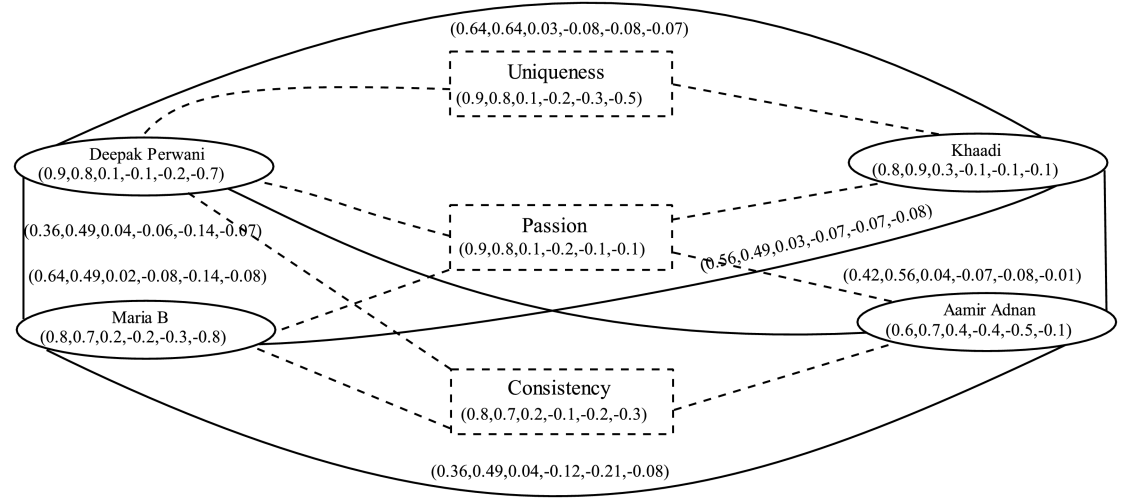

Figure 22: Corresponding bipolar single-valued neutrosophic competition graph

Table 12: Strength of competition of brands for particular quality

\begin{tabular}{|c|c|c|c|}
\hline (Brand, Quality) & In competition & $T$ (Brand, Quality) & $S$ (Brand, Quality) \\
\hline (Deepak Perwani, Uniqueness) & Khaadi & $(0.64,0.64,0.03,-0.08,-0.08,-0.07)$ & 1.04 \\
\hline (Khaadi, Uniqueness) & Deepak Perwani & $(0.64,0.64,0.03,-0.08,-0.08,-0.07)$ & 1.04 \\
\hline (Deepak Perwani, Passion) & $\begin{array}{l}\text { Khaadi, } \\
\text { Maria B, }\end{array}$ & $(0.54,0.54,0.03,-0.07,-0.12,-0.07)$ & 1.09 \\
\hline (Khaadi, Passion) & $\begin{array}{c}\text { Aamir Adnan } \\
\text { Aamir Adnan, } \\
\text { Deepak Perwani, } \\
\text { Maria B }\end{array}$ & $(0.54,0.56,0.03,-0.07,-0.07,-0.05)$ & 1 \\
\hline (Maria B, Passion) & $\begin{array}{l}\text { Khaadi, } \\
\text { Deepak Perwani, } \\
\text { Aamir Adnan }\end{array}$ & $(0.52,0.51,0.03,-0.09,-0.14,-0.05)$ & 1.08 \\
\hline (Aamir Adnan, Passion) & $\begin{array}{l}\text { Khaadi, } \\
\text { Deepak Perwani, } \\
\text { Maria B }\end{array}$ & $(0.38,0.51,0.21,-0.08,-0.14,-0.05)$ & 0.77 \\
\hline (Deepak Perwani, Consistency) & $\begin{array}{c}\text { Maria B, } \\
\text { Aamir Adnan }\end{array}$ & $(0.50,0.49,0.03,-0.07,-0.14,-0.07)$ & 1.12 \\
\hline (Maria B, Consistency) & $\begin{array}{l}\text { Deepak Perwani, } \\
\text { Aamir Adnan }\end{array}$ & $(0.50,0.49,0.03,-0.10,-0.17,-0.08)$ & 1.13 \\
\hline (Aamir Adnan, Consistency) & $\begin{array}{c}\text { Maria B, } \\
\text { Deepak Perwani }\end{array}$ & $(0.36,0.49,0.04,-0.09,-0.17,-0.07)$ & 0.99 \\
\hline
\end{tabular}

We now elaborate this method by an algorithm.

\section{Algorithm}

Step 1. Input the positive truth, indeterminacy and falsity-memberships values for set of $q$ brands.

Step 2. If for any two distinct vertices $y_{i}$ and $y_{j}, t^{p}\left(y_{i} y_{j}\right)>0, i^{p}\left(y_{i} y_{j}\right)>0, f^{p}\left(y_{i} y_{j}\right)>0$, $t^{n}\left(y_{i} y_{j}\right)<0, i^{n}\left(y_{i} y_{j}\right)<0, f^{n}\left(y_{i} y_{j}\right)<0$, then

$$
\left(y_{j}, t^{p}\left(y_{i} y_{j}\right), i^{p}\left(y_{i} y_{j}\right), f^{p}\left(y_{i} y_{j}\right), t^{n}\left(y_{i} y_{j}\right), i^{n}\left(y_{i} y_{j}\right), f^{n}\left(y_{i} y_{j}\right)\right) \in \mathcal{N}^{+}\left(y_{i}\right) .
$$

Step 3. Repeat Step 2 for all vertices $y_{i}$ and $y_{j}$ to calculate bipolar single-valued neutrosophicout-neighbourhoods $\mathcal{N}^{+}\left(y_{i}\right)$.

Step 4. Calculate $\mathcal{N}^{+}\left(y_{i}\right) \cap \mathcal{N}^{+}\left(y_{j}\right)$ for each pair of distinct vertices $y_{i}$ and $y_{j}$.

Step 5. Calculate $h\left[\mathcal{N}^{+}\left(y_{i}\right) \cap \mathcal{N}^{+}\left(y_{j}\right)\right]$. 
Step 6. If $\mathcal{N}^{+}\left(y_{i}\right) \cap \mathcal{N}^{+}\left(y_{j}\right) \neq \emptyset$ then draw an edge $y_{i} y_{j}$.

Step 7. Repeat step 6 for all pair of distinct vertices.

Step 8. Assign membership values to each edge $y_{i} y_{j}$ using the conditions

$$
\begin{array}{ll}
t^{p}\left(y_{i} y_{j}\right)=\left(y_{i} \wedge y_{j}\right) h_{1}\left[\mathcal{N}^{+}\left(y_{i}\right) \cap \mathcal{N}^{+}\left(y_{j}\right)\right] & t^{n}\left(y_{i} y_{j}\right)=\left(y_{i} \vee y_{j}\right) h_{4}\left[\mathcal{N}^{+}\left(y_{i}\right) \cap \mathcal{N}^{+}\left(y_{j}\right)\right] \\
i^{p}\left(y_{i} y_{j}\right)=\left(y_{i} \wedge y_{j}\right) h_{2}\left[\mathcal{N}^{+}\left(y_{i}\right) \cap \mathcal{N}^{+}\left(y_{j}\right)\right] & i^{n}\left(y_{i} y_{j}\right)=\left(y_{i} \vee y_{j}\right) h_{5}\left[\mathcal{N}^{+}\left(y_{i}\right) \cap \mathcal{N}^{+}\left(y_{j}\right)\right] \\
f^{p}\left(y_{i} y_{j}\right)=\left(y_{i} \vee y_{j}\right) h_{3}\left[\mathcal{N}^{+}\left(y_{i}\right) \cap \mathcal{N}^{+}\left(y_{j}\right)\right] & f^{n}\left(y_{i} y_{j}\right)=\left(y_{i} \wedge y_{j}\right) h_{6}\left[\mathcal{N}^{+}\left(y_{i}\right) \cap \mathcal{N}^{+}\left(y_{j}\right)\right]
\end{array}
$$

Step 9. If $y, r_{1}, r_{2}, r_{3}, \ldots, r_{q}$ are the brands competing for quality $d$, then strength of competition $T(y, d)=\left(t^{p}(y, d), i^{p}(y, d), f^{p}(y, d), t^{n}(y, d), i^{n}(y, d), f^{n}(y, d)\right)$ of each brand $y$ for the quality $d$ is

$T(y, d)=\frac{\left(t^{p}\left(y r_{1}\right)+\ldots+t^{p}\left(y r_{q}\right), i^{p}\left(y r_{1}\right)+\ldots+i^{p}\left(y r_{q}\right), f^{p}\left(y r_{1}\right)+\ldots+f^{p}\left(y r_{q}\right), t^{n}\left(y r_{1}\right)+\ldots+t^{n}\left(y r_{q}\right), i^{n}\left(y r_{1}\right)\right.}{q}+$ $\ldots+\frac{\left.i^{n}\left(y r_{q}\right), f^{n}\left(y r_{1}\right)+\ldots+f^{n}\left(y r_{q}\right)\right)}{q}$.

Step 10. Calculate $S(y, d)$, the strength of competition of each brand $y$ for quality $d$.

$$
S(y, d)=t^{p}(y, d)-\left(i^{p}(y, d)+f^{p}(y, d)\right)+1+t^{n}(y, d)-\left(i^{n}(y, d)+f^{n}(y, d)\right) .
$$

\section{$\S 5$ Conclusion}

Graph theory is an enjoyable playground for the research of proof techniques in discrete mathematics. There are many applications of graph theory in different fields. The notion of bipolar fuzzy graphs was first introduced by Akram in 2011 as a generalization fuzzy graphs. The idea that lies behind such description is connected with the existence of "bipolar information" (e.g., positive information and negative information) about the given set. Positive information represents what is granted to be possible, while negative information represents what is considered to be impossible. Actually, a wide variety of human decision making is based on double-sided or bipolar judgemental thinking on a positive side and a negative side. For instance, cooperation and competition, friendship and hostility, common interests and conflict of interests, effect and side effect, likelihood and unlikelihood, feedforward and feedback, and so forth are often the two sides in decision and coordination. Thus, bipolar fuzzy models indeed have potential impacts on many fields, including artificial intelligence, computer science, information science, cognitive science, decision science, management science, economics, neural science, quantum computing, medical science, and social science. In recent years bipolar fuzzy models seem to have been studied and applied a bit enthusiastically and a bit increasingly. We now have introduced the concept of the bipolar single-valued neutrosophic competition graph as a generalized structure of bipolar fuzzy graph, which gives more precision, compatibility and flexibility to a system when compared with bipolar fuzzy graphs. We aim to extend our research work to (1) Bipolar fuzzy rough graphs; (2) Bipolar fuzzy rough hypergraphs, (3) Bipolar fuzzy rough neutrosophic graphs, and (4) Decision support systems based on bipolar single-valued neutrosophic graphs. 


\section{References}

1. M Akram. Bipolar fuzzy graphs, Information Sciences, 2011, 181(24): 5548-5564.

2. M Akram. Bipolar fuzzy graphs with applications, Knowledge-Based Systems, 2013, 39: 1-8.

3. M Akram. Single-valued neutrosophic planar graphs, International Journal of Algebra and Statistics, 2016, 5(2): 157-167.

4. M Akram, M Sarwar. Novel multiple criteria decision making methods based on bipolar neutrosophic sets and bipolar neutrosophic graphs, Italian journal of pure and applied mathematics, 38(2017), 368-389.

5. M Akram, S Shahzadi. Neutrosophic soft graphs with application, Journal of Intelligent \& Fuzzy Systems, 2017, 32(1): 841-858.

6. M Akram, M Nasir. Interval-valued neutrosophic competition graphs, Annals of Fuzzy Mathematics and Informatics, 2017, 14(1): 99-120.

7. M Akram, K P Shum. Bipolar single-valued neutrosophic planar graphs, Journal of Mathematical Research with Applications, 36(6) 2017, 631-648.

8. N O Al-Shehrie, M Akram. Bipolar fuzzy competition graphs, Ars Combinatoria, 2015, 121: 385-402.

9. K T Atanassov. Intuitionistic fuzzy sets, VII ITKR's Session, Deposited in Central for Sciences Technical Library of Bulgarian Academy of Science, 1697/84, Sofia, Bulgaria, 1983.

10. P Bhattacharya. Some remark on fuzzy graphs, Pattern Recognition Letters, 1987, 6: 297-302.

11. J E Cohen. Interval graphs and food webs: a finding and a problems, Document 17696- PR, RAND Corporation, Santa Monica, CA, 1968.

12. I Deli, M Ali, F Smarandache. Bipolar neutrosophic sets and their application based on multicriteria decision making problems, In Advanced Mechatronic Systems (ICAMechS), International Conference IEEE, 2015, 249-254.

13. A Kauffman. Introduction a la theorie des sousemsembles flous, Masson et cie Paris, 1973.

14. M Nasir, S Siddique, M Akram. Novel properties of intuitionistic fuzzy competition graphs, Journal of Uncertain Systems, 2017, 2(1): 49-67.

15. S Sahoo, M Pal. Intuitionistic fuzzy competition graphs, Journal of Applied Mathematics and Computer Science, 2015, DOI 10.1007/s12190-015-0928-0.

16. S Samanta, M Pal. Fuzzy k-competition graphs and p-competition fuzzy graphs, Fuzzy Inf Eng., 2013, 5: 191-204.

17. S Samanta, M Akram, M Pal. m-Step fuzzy competition graphs, Journal of Applied Mathematics and Computing, 2015, 47: 461-472.

18. M Sarwar, M Akram. Novel concepts of bipolar fuzzy competition graphs, Journal of Applied Mathematics and Computing, 2016, DOI 10.1007/s12190-016-1021-z.

19. F Smarandache. Neutrosophic set-a generalization of the intuitionistic fuzzy set, Granular Computing, 2006, IEEE International Conference 38-42 DOI:10.1109/GRC 1635754.

20. F Smarandache. Neutrosophy, Neutrosophic probability, set and logic, American Res Press, 1998: $7-8$. 
21. F Smarandache. A geometric interpretation of the neutrosophic set- A generalization of the intuitionistic fuzzy set, Granular Computing, (GrC), 2011 IEEE International Conference, 2011, 602-606 DOI:10.1109/GRC.2011.6122665.

22. F Smarandache. Types of neutrosophic graphs and neutrosophic algebraic structures together with their applications in technology, Seminar, Universitatea Transilvania din Brasov, Facultatea de Design de Produs si Mediu, Brasov, Romania, 06 June 2015.

23. F Smarandache. A unifying field in logics: neutrosophic logic. neutrosophy, meutrosophic set, neutrosphic probability: neutrosophic logic. neutrosophy, neutrosophic set, neutrosophic prbability, Inifinite Study, 2005.

24. H Wang, F Smarandache, Y Zhang, R Sunderraman. Single valued neutrosophic sets, Multisapace and Multistructure, 2010, 4: 410-413.

25. S Y Wu. The compositions of fuzzy digraphs, Journal of Research in Education Science,1986, 31: 603-628.

26. J Ye, Single-valued neutrosophic minimum spanning tree and its clustering method, Journal of Intelligent Systems, 2014, 23(3): 311-324.

27. J Ye. Improved correlation coefficients of single-valued neutrosophic sets and interval neutrosophic sets for multiple attribute decision making, Journal of Intelligent \& Fuzzy Systems, 2014, 27(5): 2453-2462.

28. J Ye. A multicriteria decision-making method using aggregation operators for simplified neutrosophic sets, Journal of Intelligent \& Fuzzy Systems, 2014, 26(5): 2459-2466.

29. J Ye. Vector similarity measures of simplified neutrosophic sets and their application in multicriteria decision making, International Journal of Fuzzy Systems, 2014, 16(2).

30. J Ye. Improved cosine similarity measures of simplified neutrosophic sets for medical diagnoses, Artificial intelligence in medicine, 2015, 63(3): 171-179.

31. J Ye. Single valued neutrosophic cross-entropy for multicriteria decision making problems, Applied Mathematical Modeling, 2014, 38(3) : 1170-1175.

32. J Ye. Clustering methods using distance-based similarity measures of single-valued neutrosophic sets, Journal of Intelligent Systems, 2014, 23(4): 379-389.

33. L A Zadeh. Fuzzy sets, Information and Control, 1965, 08: 338-353.

34. L A Zadeh. Similarity relations and fuzzy orderings, Information Science, 1971, 3(2), (1971): 177-200.

35. W -R Zhang. Bipolar fuzzy sets and relations: a computational framework for cognitive modeling and multiagent decision analysis, In: Proceedings of IEEE Conference Fuzzy Information Processing Society Biannual Conference, 1994: 305-309.

${ }^{1}$ Department of Mathematics, University of the Punjab, New Campus, Lahore, Pakistan.

Email: m.akram@pucit.edu.pk, maryamnasir912@gmail.com

${ }^{2}$ Institute of Mathematics, Yunnan University, China

Email: kpshum@ynu.edu.cn 